\title{
Cosmology of codimension-two braneworlds
}

\author{
James M. Cline ${ }^{1,2}$, Julie Descheneau, ${ }^{2}$, Massimo Giovannini ${ }^{1}$, Jérémie Vinet $^{2}$ \\ 1 Theory Division, CERN CH-1211, Geneva 23, Switzerland \\ 2 Physics Department, McGill University, 3600 University Street, Montréal, Québec, \\ Canada H3A 2 T8 \\ E-mail: James.Cline@cern.ch, jdesch@hep.physics.mcgill.ca, \\ Massimo.Giovannini@cern.ch, vinetj@physics.mcgill.ca
}

\begin{abstract}
We present a comprehensive study of the cosmological solutions of $6 \mathrm{D}$ braneworld models with azimuthal symmetry in the extra dimensions, moduli stabilization by flux or a bulk scalar field, and which contain at least one 3-brane that could be identified with our world. We emphasize an unusual property of these models: their expansion rate depends on the 3-brane tension either not at all, or in a nonstandard way, at odds with the naive expected dimensional reduction of these systems to $4 \mathrm{D}$ general relativity at low energies. Unlike other braneworld attempts to find a self-tuning solution to the cosmological constant problem, the apparent failure of decoupling in these models is not associated with the presence of unstabilized moduli; rather it is due to automatic cancellation of the brane tension by the curvature induced by the brane. This provides some corroboration for the hope that these models provide a distinctive step toward understanding the smallness of the observed cosmological constant. However, we point out some challenges for obtaining realistic cosmology within this framework.
\end{abstract}




\section{Contents}

1. Introduction 1

2. Bulk Solutions 3

2.1 Type 1 solutions

2.2 Type 2 solutions

2.3 Type 3 solutions 8

3. Brane tensions and jump conditions 9

3.1 Jump condition at $\rho=0$

3.2 Metric jump conditions at $\rho=\rho_{m}$

3.3 Jump conditions for $\phi \quad 11$

4. Stabilization in warped model 11

4.1 Stabilization by bulk scalar 11

4.2 Back reaction of scalar (or magnetic flux) on metric 12

4.3 Solution of jump conditions 13

4.4 Stabilization by magnetic flux? 14

5. Friedmann equation in warped model 15

6. Stability of unwarped model 18

7. Discussion and conclusions 22

A. Perturbation to AdS soliton from small $\Lambda_{4}$

B. Cosmology of codimension one branes in six dimensions 25

B.1 Boundary conditions 25

$\overline{\mathrm{B} .2}$ Order $\left(\rho^{0}\right)$

B.3 Order $\rho \quad 26$

\section{Introduction}

The cosmology of codimension one branes, notably 3-branes in a 5D universe, has been intensively studied and is now rather well understood. One of the striking predictions of this picture is that the Friedmann equation for the Hubble expansion rate should have the form $H^{2}=\frac{8 \pi}{3} G \rho\left(1+\frac{\rho}{T}\right)$ in the simplest situation with a single brane of tension $T$ and excess energy density $\rho$, in a warped space where the bulk is $5 \mathrm{D}$ anti-deSitter space [1]-[3]. 
One naturally wonders whether this kind of behavior is particular to branes of codimension one (i.e., having only one transverse dimension), or if higher codimension branes can act similarly. There have been numerous attempts over the last twenty years to harness some of the unusual features of six dimensional models in order to get some insight into the cosmological constant problem [4]-14], the weak scale hierarchy problem [5, 6], 15]-22] or other effects of warped compactification [23]-28], and moduli stabilization [22], 229]- 32]. However we are not aware of any attempts to systematically explore modifications to the Friedmann equation in codimension two braneworld models. In fact, most of the work referred to above has been confined to the study of static solutions.

In this paper we consider the most symmetric case, where the extra dimensions have azimuthal symmetry and the metric functions depend only upon the radial coordinate of the bulk, with periodic angular coordinate $\theta \in[0,2 \pi]$ :

$$
d s^{2}=M^{2}(\rho) g_{\mu \nu} d x^{\mu} d x^{\nu}+d \rho^{2}+L^{2}(\rho) d \theta^{2}
$$

here $g_{\mu \nu}$ is a maximally symmetric $4 \mathrm{D}$ metric which can be deSitter, anti-deSitter, or Minkowski space, with cosmological constant $\Lambda_{4}$, normalized such that the scale factor is $a(t)=e^{H t}$ with $H=\kappa_{6} \sqrt{\Lambda_{4} / 3}$ in terms of the $6 \mathrm{D}$ gravitational constant $\kappa_{6}$. General FRW solutions in the $4 \mathrm{D}$ spacetime would require having distinct warp factors for $d t^{2}$ and $\left(d x^{i}\right)^{2}$, a complication which we choose to avoid in the present study. We will assume that the only bulk contributions to the stress energy are a $6 \mathrm{D}$ cosmological constant $\Lambda_{6}$, a magnetic flux $F_{\rho \theta}$, or a bulk scalar field $\phi$.

In section 2 we will classify all the possible bulk solutions which are consistent with (1.1) and point out the insensitivity of $\Lambda_{4}$ to 3 -brane tensions in some of these solutions, including a particularly novel one in section 2.1. In section 3 we discuss the boundary conditions associated with the tensions of branes which bound these solutions, which are the source of any dependence of the Hubble rate on the brane tensions which does exist. Section 4 sets the stage for investigating the Friedmann equation in a class of warped solutions by reviewing how these solutions can be stabilized by a bulk scalar field. This solution is known as the AdS soliton, since it looks like $\mathrm{AdS}_{6}$ at large radii. In section 5 we derive a surprising result for the AdS soliton, namely that the Friedmann equation relating the expansion rate to the brane tensions has an unusual form which does not agree with the expectation that the system should reduce to $4 \mathrm{D}$ general relativity at distances much larger than the compactification scale. In section 6 we turn our attention to an unwarped solution, studied recently in [13, 14], where the bulk is a two-sphere stabilized by magnetic flux and deformed by deficit angles due to 3-branes at antipodal points. We look for unstable modes in the spectrum of fluctuations around this solution which might explain why the expansion rate of these models is insensitive to the 3-brane tensions, and show that no such modes exist. We conclude with a discussion of how $2 \mathrm{D}$ compactification manifolds manage to tune away the effects of 3-brane tensions in certain cases, and the difficulties that will have to be overcome if one wants to study realistic cosmology by putting matter with a general equation of state on the branes. 


\section{Bulk Solutions}

We start by writing the Einstein and scalar field equations for the metric (1.1). Defining $\mu=M^{\prime} / M, \ell=L^{\prime} / L$, and working in units where the $6 \mathrm{D}$ gravitational constant $\kappa_{6}^{2}=1$, these equations are

$$
\begin{aligned}
\mu \mu: \quad \ell^{\prime}+3 \mu^{\prime}+\ell^{2}+6 \mu^{2}+3 \ell \mu & =-\Lambda_{6}+\frac{\Lambda_{4}}{M^{2}}-\frac{1}{2}\left(\phi^{\prime 2}+m^{2} \phi^{2}+\frac{n^{2} \phi^{2}}{L^{2}}\right)-\frac{\beta^{2}}{2 M^{8}} \\
\theta \theta: \quad 4 \mu^{\prime}+10 \mu^{2} & =-\Lambda_{6}+\frac{2 \Lambda_{4}}{M^{2}}-\frac{1}{2}\left(\phi^{\prime 2}+m^{2} \phi^{2}-\frac{n^{2} \phi^{2}}{L^{2}}\right)+\frac{\beta^{2}}{2 M^{8}} \\
\rho \rho: 4 \mu \ell+6 \mu^{2} & =-\Lambda_{6}+\frac{2 \Lambda_{4}}{M^{2}}+\frac{1}{2}\left(\phi^{\prime 2}-m^{2} \phi^{2}-\frac{n^{2} \phi^{2}}{L^{2}}\right)+\frac{\beta^{2}}{2 M^{8}} \\
\phi: \quad \phi^{\prime \prime}+(4 \mu+\ell) \phi^{\prime} & =m^{2} \phi+\frac{n^{2} \phi^{2}}{L^{2}}
\end{aligned}
$$

The $4 \mathrm{D}$ cosmological constant is defined by $H^{2}=\Lambda_{4} / 3$ in the de Sitter brane case, where the $4 \mathrm{D}$ line element has the FRW form $d s^{2}=-d t^{2}+e^{2 H t} d \vec{x}^{2}$ We have allowed for the scalar field to be either real or complex with a winding number $n$. The constant $\beta$ is related to the magnetic field strength by $F_{\rho \theta}=\beta L / M^{4}$, which can be seen to satisfy the Maxwell equation $\partial_{A}\left(\sqrt{|G|} F^{A B}\right)=0$. For simplicity we have assumed that the bulk scalar has no self interactions, so $V(\phi)=\frac{1}{2} m^{2} \phi^{2}$. Only three of these equations are independent; for example the $(\mu \mu)$ equation can be derived from differentiating $(\rho \rho)$ and combining with the other equations.

Rubakov and Shaposhnikov [ [⿴囗十 found an elegant way to solve the 6D Einstein equations resulting from the metric (1.1), which was generalized to include bulk magnetic flux in 5] (see also [6]). We extend the method to take into account the possible presence of bulk scalar fields. The Einstein equation (2.2) for this system is equivalent to the equation governing the classical motion of a particle of unit mass, whose action is

$$
S=\int d \rho\left(\frac{1}{2} z^{\prime 2}-U(z)-U_{\phi}(z)\right)
$$

Here $z^{\prime}=\frac{d z}{d \rho}$ and the potential depends on the $4 \mathrm{D}$ and $6 \mathrm{D}$ cosmological constants and the magnetic field via ${ }^{1}$

$$
\begin{aligned}
U(z) & =a z^{2}-b z^{6 / 5}+c z^{-6 / 5} ; \quad a=\frac{5}{16} \Lambda_{6} ; \quad b=\frac{25}{24} \Lambda_{4} ; \quad c=\frac{25}{96} \beta^{2} \\
U_{\phi}(z) & =\frac{5}{32} z^{2}\left(\phi^{\prime 2}+m^{2} \phi^{2}-\frac{n^{2} \phi^{2}}{L^{2}}\right)
\end{aligned}
$$

The radial coordinate $\rho$ plays the role of time in this analogy. If we ignore the bulk scalar $\phi$, the resulting equation of motion $z^{\prime \prime}+U^{\prime}(z)=0$ allows one to solve for the warp factor $M(\rho)$ independently of any other fields in the problem, using the relation

$$
M(\rho)=z^{2 / 5}(\rho) .
$$

\footnotetext{
${ }^{1}$ we correct a factor of 2 error in $b$ relative to 4
} 
If $\phi$ is nonzero, this is no longer possible; however one can find an approximate solution iteratively by first finding $M(z)$ when $\phi=0$, next solving for $\phi$ in the background geometry, and then finding the approximate form of the back reaction of $\phi$ on the geometry by treating $U_{\phi}$ as a perturbation.

The above procedure has so far yielded only the metric component $M$. To obtain $L$, we can in general solve the first order $(\rho \rho)$ equation; but in most cases there is a much simpler relation which gives either an exact expression or a good approximation to $L$ in terms of $M$ :

$$
L(\rho)=R \frac{d M}{d \rho} .
$$

Here $R$ is a constant of integration, which as we will show later is determined by the tension of a 3-brane which may be located at $\rho=0$. This result follows from the difference of the $(\theta \theta)$ and $(\rho \rho)$ equations, which can be written as

$$
\frac{L^{\prime}}{L}=\frac{M^{\prime \prime}}{M^{\prime}}+\frac{\phi^{2}}{4 \mu}
$$

Thus the relation $(2.9)$ is exact whenever $\phi^{\prime}=0$, with the exception of unwarped solutions where $M^{\prime}=0$. For these the $(\rho \rho)$ Einstein equation provides no information about $L$, and we must turn to the $(\mu \mu)$ equation, as will be discussed in section 2.3 .

Let us now focus on situations in which $\phi^{\prime}$ is negligible and the scalar field has no winding; in this case $U_{\phi}$ can be absorbed into the $a$ term of $U$. The potential $U(z)$ has eight possible distinctive shapes depending on the signs of $\mu$ and $\Lambda$, and whether or not there is a magnetic flux. These are summarized in figure 1 . The solutions can be visualized by starting the particle at rest $\left(z^{\prime}=0\right)$ at position $\rho=0$ with some initial value $z_{0}$, and letting it roll in the potential. The position $\rho$ should be thought of as time in the mechanics analogy. The initial condition that $z^{\prime}=0$ implies that $M^{\prime}=0$ at $\rho=0$, which must be the case; there is no singular source term for $M^{\prime \prime}$ in the Einstein equations, and therefore $M^{\prime}$ must be continuous at the origin. Considering $M^{\prime}$ evaluated along a straight line passing through the origin, one sees that continuity of the slope requires that $M^{\prime}$ in fact vanishes. Using the mechanics analogy, a closed form solution for $M(\rho)$ is implicitly provided by integrating the equation of energy conservation, $E=\frac{1}{2} z^{\prime 2}+U(z)$ :

$$
\rho=\left|\int_{z_{0}}^{z} \frac{d z}{\sqrt{2(E-U(z))}}\right|
$$

Once the particle starts rolling, there are four possible outcomes, which depend on the shape of the potential and the initial value $z_{0}$.

1. In one kind of solution, the particle can reach $z=0$ at some finite value of $\rho$. This happens when $\beta=0, \Lambda_{6}>0$ and $E>0$ (fig. 1a), or when $\beta=0, \Lambda_{6}<0, \Lambda_{4}<0$ and $z<z_{m}$ (fig. 1b). Some curvature invariants diverge, so the extra dimensions end at a singularity with the topology of $S_{1}$ in this case [23].

2. Another kind is where $z \rightarrow \infty$ as $\rho \rightarrow \infty$, as occurs if $\Lambda_{6}<0$ and $\Lambda_{4} \geq 0$, or if $\Lambda_{6}<0, \Lambda_{4}<0$ and $z>z_{m}$. Then gravity will not be localized, and the world does 

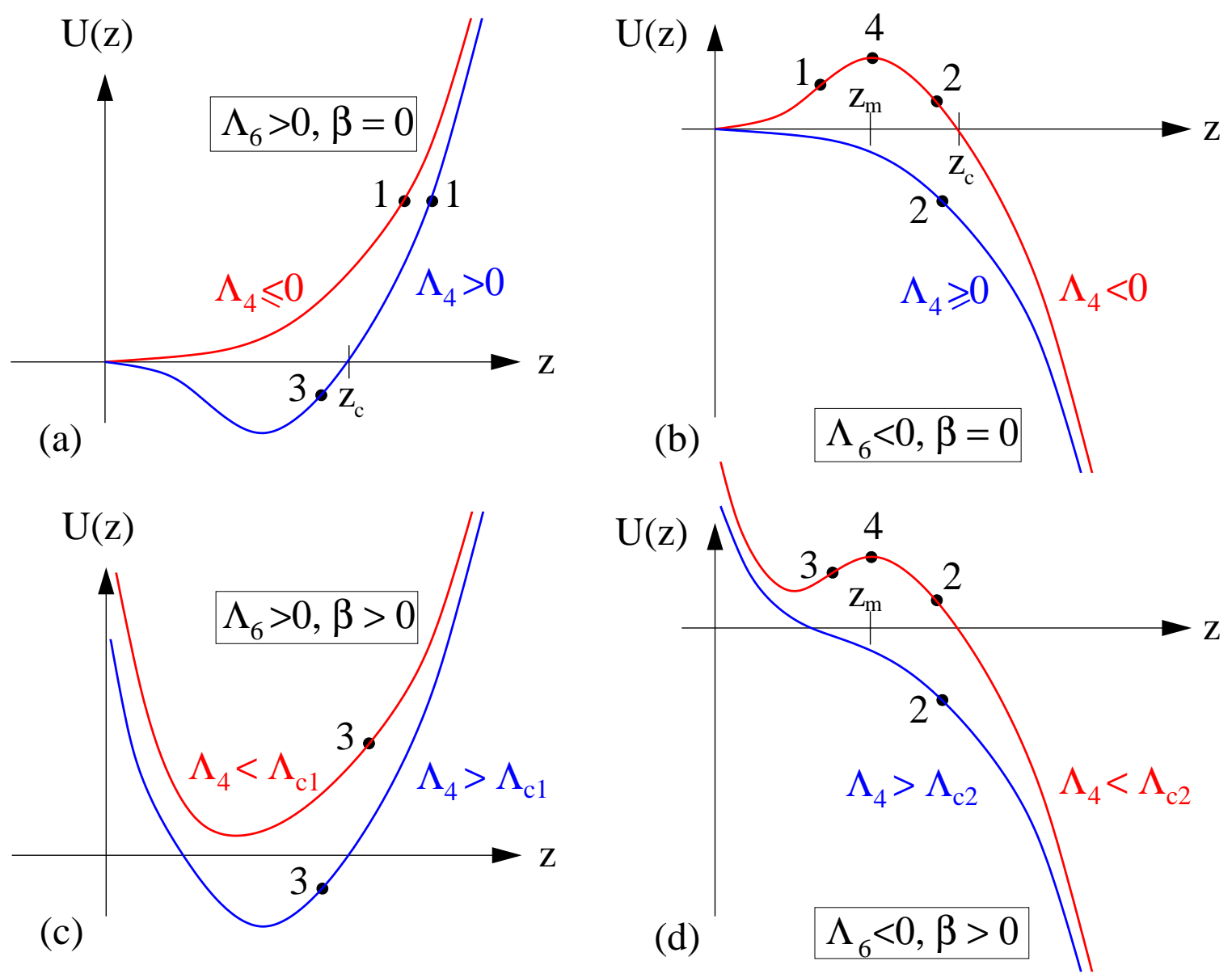

Figure 1: Shapes of the potential $U(z)$ depending on the sign of $\Lambda_{4}$ for (a) $\Lambda_{6}>0$ and (b) $\Lambda_{6}<0$ in the case of vanishing magnetic field $\beta$, and similarly in (c) and (d) for nonzero $\beta$. Numbered dots show initial conditions leading to the four kinds of solutions discussed below. The first critical value is given by $\Lambda_{c 1}^{4}=\left(2 \Lambda_{6} / 5\right)^{3} \beta^{2}$.

not look 4-dimensional to an observer on the brane. A special case of this kind is the AdS soliton solution [33], where $\beta=\Lambda_{4}=0$.

3. A third possibility is that the particle starts to the left of a stable equilibrium position, in which case it comes to rest again at the other side of the minimum at some maximum value of $\rho=\rho_{m}$. The vanishing of $z^{\prime}$ means that the space closes off at $\rho_{m}$, and we can consistently insert a second 3 -brane at this position. The extra dimensions look like $S_{2}$ deformed by deficit angles due to the two 3-branes at antipodal points. A special case [13, 14] is when the particle stays at rest at the equilibrium position. This gives an unwarped solution since $M(\rho)$ remains constant. Nonvanishing magnetic flux $\beta$ is required to obtain static solutions of this kind.

4. One can also find special unwarped solutions when the particle sits at an unstable equilibrium position, denoted in fig. 1 by $z_{m}$, in the case of an AdS 3-brane. Since 
we are interested in cosmological solutions, we do not pursue these here.

To cure the problems of cases (1) and (2) above, one should cut out the part of the space with $z=0$ or $z=\infty$ at some value $z=z_{4}$, and insert a 4 -brane at the corresponding radius $\rho=\rho_{m}[22]$. In fact it is not generally possible to satisfy the jump conditions (to be described in section 3) at $\rho_{m}$ using an ordinary 4-brane, since the bulk solution requires that its stress-energy components $T_{\theta \theta}$ differ from the other spatial components $T_{i i}$. One way of accomplishing this is to imagine that a pure tension 4-brane is accompanied by a 3-brane which is smeared around the compact extra dimension [34], or by Casimir energy of a massless field confined to the 4-brane [17]. Alternatively, it is possible to find a special value of $\rho_{m}$ where the jump conditions on $M$ and $L$ can both be satisfied for a pure tension 4-brane, as long as $\Lambda_{4}$ is nonzero. By integrating the exact result 4.10) which we will derive in section 4.3 , this value is implicitly given by

$$
\int_{0}^{\rho_{m}} M^{2} L d \rho=\frac{L^{\prime}(0)}{\Lambda_{4}}
$$

showing that $\rho_{m} \rightarrow \infty$ as $\Lambda_{4} \rightarrow 0$ for the warped solutions where $M$ and $L$ grow exponentially. If one wants a solution which remains compact in the static limit, this is not acceptable.

For completeness we mention one other kind of solution: we can start with $z^{\prime} \neq 0$ at $\rho=0$, but in this case the position $\rho=0$ should not be regarded as a single point, but rather the location of another 4-brane. A special case is when $\Lambda_{6}<0, \Lambda=E=0$; then the warp factor takes the simple exponential form of the RS model. In fact this is just the 5D RS solution augmented by one extra compact dimension, whose warp factor is identical to that of the large dimensions. This model does not involve 3 -branes, and is mathematically quite similar to the $5 \mathrm{D}$ case which has been so well studied already. A more thorough examination of the cosmology of these solutions in presented in appendix $\mathrm{B}$, and we comment upon its qualitative differences relative to the codimension two case in the conclusions.

In the following subsections we present some specific analytic solutions illustrating the cases mentioned above.

\subsection{Type 1 solutions}

A solution which is borderline between type 1 and type 3 can be found by choosing $\Lambda_{4}, \Lambda_{6}>$ $0, E=0$. Starting from $z=z_{c}=(b / a)^{5 / 4}$, the critical value shown in fig. 1(a) defined by $U\left(z_{c}\right)=0$, the particle subsequently rolls toward $z=0$. The solution is

$$
M(\rho)=\cos (k \rho) ; \quad L(\rho)=R k \sin (k \rho) ; \quad k=\sqrt{\frac{\Lambda_{6}}{10}}=H
$$

where we have used the freedom to rescale $x^{\mu}$ to make $M(0)=1$. Recall that $H=\sqrt{\Lambda_{4} / 3}$ is the Hubble constant, so that $\Lambda_{4}$ is determined by

$$
\Lambda_{4}=\frac{3}{10} \Lambda_{6}
$$


Interestingly, no curvature invariants diverge at the point $\rho_{m}=\pi / 2 k$ where $M=0$ and the space should be terminated. In fact the Ricci scalar is constant, $R=3 \Lambda_{6}$, and so are the Ricci tensor squared $R_{\alpha \beta} R^{\alpha \beta}=\frac{3}{2} \Lambda_{6}^{2}$, and Riemann tensor squared $R_{\alpha \beta \gamma \delta} R^{\alpha \beta \gamma \delta}=\frac{3}{5} \Lambda_{6}^{2}$. The Weyl tensor vanishes. This is analogous to the behavior as $y \rightarrow \infty$ in the 5D RandallSundrum solution [35] with the line element $d s^{2}=e^{-2 k|y|}\left(-d t^{2}+d \vec{x}^{2}\right)+d y^{2}$; even though the warp factor vanishes as $y \rightarrow \infty$ in this model, there is no singularity. Although $M^{\prime}\left(\rho_{m}\right) \neq 0$, yet $z^{\prime}=5 / 2 M^{3 / 2} M^{\prime}$ vanishes at $\rho_{m}$, so in the mechanics analogy, the ball rolls to the top of the hill and comes to rest again. Apparently the $4 \mathrm{D}$ part of the spacetime disappears at $\rho_{m}$, leaving one with just 2D Euclidean space in the $\rho-\theta$ plane.

In the case of vanishing $\Lambda_{4}$ and positive $\Lambda_{6}$, the following solution can be found [9]

$$
M(\rho)=\cos (k \rho)^{2 / 5} ; \quad L(\rho)=R k \sin (k \rho)^{2 / 5} ; \quad k=\sqrt{\frac{5}{8} \Lambda_{6}} .
$$

In this case however, in the mechanics analogy the particle does not come to rest at $z=0$, and there will be a curvature singularity at that point. It is therefore necessary to insert a 4-brane at $\rho<\rho_{s}$ to cut off the space before reaching the point where it becomes singular.

\subsection{Type 2 solutions}

It is interesting to consider solutions with $\Lambda_{6}<0$ since this gives a warped geometry, where the hierarchy problem can be solved on the 3-brane $\grave{a}$ la Randall-Sundrum. We find a solution of type 2 when $\beta=E=0$ and $\Lambda_{4}, \Lambda_{6}<0$ by analytically continuing (2.13) in $\Lambda_{4}$ and $\Lambda_{6}$ :

$$
M(\rho)=\cosh (k \rho) ; \quad L(\rho)=R k \sinh (k \rho) ; \quad k=\sqrt{\frac{\left|\Lambda_{6}\right|}{10}}=i H
$$

This follows the upper curve of fig. 1(b), starting at $z_{c}$. The Hubble constant is imaginary because the $4 \mathrm{D}$ metric is AdS space. In addition the $E=0$ type 2 solution with $\Lambda_{4}>0$ and $\Lambda_{6}<0$ is

$$
M(\rho)=\sinh (k \rho) ; \quad L(\rho)=R k \cosh (k \rho)
$$

which follows the lower curve of fig. 1(b), starting at $z=0$. However, this solution does not admit a 3 -brane at $\rho=0$ since $L$ is nonvanishing at this point.

The only way to admit a 3-brane at $\rho=0$ if $\Lambda_{4} \geq 0$ is to take $E<0$, so that the initial condition $z^{\prime}=0$ (equivalent to the boundary condition $M^{\prime}=0$ ) can be satisfied. The static solution with $\Lambda_{4}=0$ (and $\beta=0$ ) has been investigated in 34, 22]; this is the AdS soliton truncated at large $\rho$ by a 4 -brane:

$$
M(\rho)=\cosh ^{\frac{2}{5}}(k \rho) ; \quad L(\rho)=\frac{2}{5} R k \frac{\sinh (k \rho)}{\cosh ^{\frac{3}{5}}(k \rho)} \quad k=\sqrt{-\frac{5}{8} \Lambda_{6}}
$$

Analytic expressions for $M(\rho)$ in the AdS soliton type of solution no longer exist when $\Lambda_{4}>0$. However, we can obtain approximate analytic solutions by considering $\Lambda_{4}$ as a perturbation. The details are given in appendix A. The result is that the unperturbed solution gets shifted according to

$$
M(\rho)=\left[z_{0} \cosh (k(\rho+\delta \rho))\right]^{2 / 5} ; \quad L(\rho)=R M^{\prime}(\rho)
$$


where $\delta \rho(z)$ has the shape shown in fig. 2, and $z=z_{0} \cosh (k \rho)$. At sufficiently large values of $\rho$, the shift $\delta \rho$ approaches a constant, so at leading order the only difference between the perturbed and unperturbed solutions at large $\rho$ is a shift in the radial size of the extra dimension.

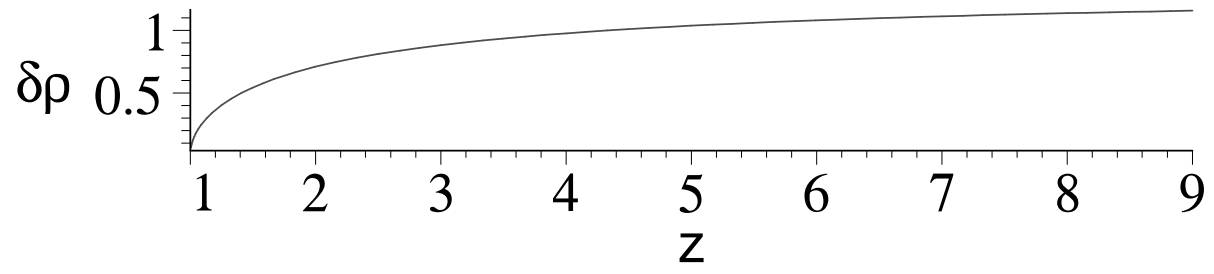

Figure 2: $\delta \rho$ versus $z$, due to nonzero $\Lambda_{4}$ in the AdS soliton solution.

\subsection{Type 3 solutions}

The simplest example of a solution of type 3 is that in which the particle sits at a minimum of $U$. In this case $M$ is constant, so the solution is unwarped. If there is no magnetic field, this occurs only in fig. 1(a), when $\Lambda_{4}>0$. To specify the physical value of $\Lambda_{4}$, we should rescale $M \rightarrow 1$ so that time and energy are normalized in the usual way $\left(d s^{2}=\right.$ $-d t^{2}+e^{2 H t} d \vec{x}^{2}$ ). Since $\Lambda_{4}=3 H^{2}$, which has dimensions of (mass) ${ }^{2}$, the relevant quantity is $\Lambda_{4} / M^{2}=\Lambda_{6} / 2$; in other words the expansion rate is governed completely by $\Lambda_{6}$, which is to be expected since it is the only source of stress energy.

Another example of unwarped solutions is the static case $\Lambda_{4}=0$ with nonvanishing magnetic field. Just like above we noted that the physically relevant combination for the expansion rate is $\Lambda_{4} / M^{2}$, in this case $\beta^{2} / M^{8}$ is the meaningful combination for the square of the magnetic field strength. Minimizing $U(z)$ we find that $\beta^{2} / M^{8}=2 \Lambda_{6}$, in agreement with references [13, 14]. Notice that $\Lambda_{6}$ must be positive to achieve this fine tuning with the magnetic flux.

In fact, the general relation between $\Lambda_{4}, \Lambda_{6}$ and $\beta$ can be easily found in the same way, demanding $U^{\prime}\left(z_{0}\right)=0$, when we realize that there is the freedom to set $z_{0} \rightarrow 1$ by rescaling $x_{\mu} \rightarrow x_{\mu} / z_{0}$. Thus

$$
\Lambda_{4}=\frac{1}{2} \Lambda_{6}-\frac{1}{4} \beta^{2}
$$

characterizes the physical expansion rate for an observer on the 3-brane when the bulk parameters are not tuned to give a static solution. This is a significant result because it is completely independent of the tension of such a 3-brane 13, 14.

Although $M$ is trivial for the unwarped solutions, $L$ is nontrivial:

$$
L(\rho)=R \sin (k \rho) ; \quad k^{2}=\frac{1}{2} \Lambda_{6}+\frac{1}{4} \beta^{2}
$$

as follows from solving the $(\mu \mu)$ Einstein equation (2.1), using (2.19) to eliminate $\Lambda_{4}$.

More general solutions of type 3 cannot be found exactly, but an approximation is possible for values of $E$ which are sufficiently close to the bottom of the potential in fig. 
1(a) so that it can be treated like a harmonic oscillator. This gives

$$
M(\rho) \cong\left(z_{0}+\epsilon \cos (k \rho)\right)^{2 / 5}
$$

where $z_{0}$ is the position of the minimum of the potential, and $k^{2}=U^{\prime \prime}\left(z_{0}\right)$. In general $k$ and $\epsilon$ are functions of $\Lambda_{6}, \Lambda_{4}$ and $\beta$. For example, in the case without magnetic flux, $z_{0}=\left(2 \Lambda_{4} / \Lambda_{6}\right)^{5 / 4}$ and $k^{2}=\frac{1}{2} \Lambda_{6}$. If one wants to rescale the warp factor to unity, the physically meaningful place to do so is on the brane where the observer is supposed to be, for example $z_{0}+\epsilon \rightarrow 1$.

Interestingly, in the more general solution (2.21), some dependence of the expansion rate on the tensions $\tau_{3}, \tau_{3}^{\prime}$ of the two 3-branes appears. An application of the jump conditions to be described in section 3.1 gives the following constraint:

$$
\frac{\tau_{3}-2 \pi}{\tau_{3}^{\prime}-2 \pi}=\left(\frac{z_{0}-\epsilon}{z_{0}+\epsilon}\right)^{3 / 2}
$$

This can be regarded as a fine tuning between the brane tensions required to obtain a given value of the expansion rate determined by $\Lambda_{4}$. There is a one-parameter family of brane tensions which preserve the necessary relationship, showing that the expansion rate is not uniquely determined by the brane tensions. However the degeneracy of solutions along this curve in the $\tau_{3}-\tau_{3}^{\prime}$ plane does not constitute a solution to the cosmological constant problem, and in this respect we disagree with the interpretation of [5]. In that reference, it was assumed that a deficit angle could appear spontaneously at the position of one of the branes, without having to specify any brane tension in the input to the construction of the model, namely the stress-energy tensor. Our point of view is that the 3 -brane is not created by the geometry, but rather any singularity in the $2 \mathrm{D}$ curvature is due to the presence of a 3-brane with nonvanishing tension. (Another difference between our work and that of [5] is that we do not assume the deficit angle, hence brane tension, to be zero at $\rho=0$. This enlarges the class of solutions we are considering.)

\section{Brane tensions and jump conditions}

In the previous section we generalized the analysis of [4] to include extra sources of stress energy and to consider all possible signs of these various sources. A further new ingredient which 㑑 did not consider is the presence of branes which bound the bulk solutions given above. In each case we wish to allow for a 3-brane at the origin of the two extra dimensions, $\rho=0$, which could provide support for the standard model and our observable $4 \mathrm{D}$ spacetime. (A special case is a 3-brane with vanishing tension, which is the same as no brane as far as Einstein's equations are concerned.) The space will either terminate with another 3 -brane or a 4 -brane at some maximum value $\rho_{m}$, or it may be noncompact, according to the possiblities mentioned in section 2, although the latter situation is not compatible with recovering $4 \mathrm{D}$ gravity at large distances.

\subsection{Jump condition at $\rho=0$}

In all cases, the constant of integration $R$ in (2.9) is related to the tension $\tau_{3}$ of the 3brane at $\rho=0$. If $\tau_{3}$ is nonzero, there is a conical defect with deficit angle $\delta$ given by 
$\delta=\kappa_{6}^{2} \tau_{3}$, where $\kappa_{6}^{2}=8 \pi G_{6}$, in terms of the $6 \mathrm{D}$ Newton's constant. We can compute the ratio of the circumference and the radius of an infinitesimal circle around the origin as $\lim _{\epsilon \rightarrow 0} 2 \pi L(\epsilon) / \epsilon=2 \pi L^{\prime}(0)$. Therefore, continuing to work in units where $\kappa_{6}=1$, we have

$$
\tau_{3}=2 \pi\left(1-L^{\prime}(0)\right)
$$

If the space is terminated by another 3 -brane at $\rho_{m}$ with tension $\bar{\tau}_{3}$, the relation becomes

$$
\bar{\tau}_{3}=2 \pi\left(1+L^{\prime}\left(\rho_{m}\right)\right)
$$

\subsection{Metric jump conditions at $\rho=\rho_{m}$}

On the other hand, if there is a 4 -brane at $\rho_{m}$, it may need to be accompanied by some additional form of stress energy in order to cut the bulk solution at this particular location, because there are separate jump conditions for the $\theta \theta$ and $\mu \mu$ components of the Einstein equations. Therefore one generally needs two tunable parameters to satisfy both conditions. These conditions can be inferred from looking at the terms in the Einstein equations which have second derivatives or delta functions:

$$
\begin{array}{ll}
\mu \mu: & \frac{M^{\prime \prime}}{M}+\frac{1}{3} \frac{L^{\prime \prime}}{L}+\frac{M^{\prime} L^{\prime}}{M L}+\frac{M^{\prime 2}}{M^{2}}=-\frac{1}{3} T_{0}^{0} \delta\left(\rho-\rho_{m}\right)+\ldots \\
\theta \theta: & 2 \frac{M^{\prime 2}}{M^{2}}+\frac{4 M^{\prime \prime}}{3 M}=-\frac{1}{3} T_{\theta}^{\theta} \delta\left(\rho-\rho_{m}\right)+\ldots
\end{array}
$$

We will impose $Z_{2}$ symmetry at the 4 -brane so that the discontinuity in $M^{\prime}$ is $-2 M^{\prime}$. Integrating in the vicinity of the delta functions, we find

$$
T_{0}^{0}=6 \frac{M^{\prime}}{M}+2 \frac{L^{\prime}}{L} ; \quad T_{\theta}{ }^{\theta}=8 \frac{M^{\prime}}{M}
$$

where the functions are all evaluated at $\rho_{m}$. If the stress energy tensor was given by a brane with pure tension, we would have $T_{0}{ }^{0}=T_{\theta}{ }^{\theta}$. This does not occur in the static AdS soliton solution (2.17), where $M^{\prime} / M-L^{\prime} / L$ is always nonzero, except as $\rho \rightarrow \infty$. One way to obtain a difference between $T_{0}{ }^{0}$ and $T_{\theta}{ }^{\theta}$ is to "smear" a 3-brane along the compact dimension of the 4-brane [34]. Since the 3 -brane has $T_{\theta}{ }^{\theta}=0$, this procedure will give an extra contribution only to the $T_{\mu}{ }^{\mu}$ components. The shift in $T_{0}{ }^{0}$ due to the smeared 3 -brane tension $\tau_{3}^{\prime}$ is given by $T_{0}{ }^{0}-T_{\theta}{ }^{\theta}=\tau_{3}^{\prime} /\left[2 \pi L\left(\rho_{m}\right)\right]$. This is not the only way to obtain a deviation $T_{0}{ }^{0}-T_{\theta}{ }^{\theta}$; for example the Casimir energy of a massless field confined to the 4-brane gives $T_{0}{ }^{0}-T_{\theta}{ }^{\theta} \sim L^{-5}$ [17]. In general, the energy density of the source $\tau^{\prime}$ can scale like $1 / L^{\alpha}\left(\rho_{m}\right)$, where $\alpha$ determines its equation of state. Conservation of stress-energy then dictates its contributions to the different components of the stress tensor [9], 22]:

$$
\begin{aligned}
& T_{0}{ }^{0}=T_{4}+V_{m}(\phi)+\frac{\tau^{\prime}}{L^{\alpha}\left(\rho_{m}\right)} \\
& T_{\theta}{ }^{\theta}=T_{4}+V_{m}(\phi)+(1-\alpha) \frac{\tau^{\prime}}{L^{\alpha}\left(\rho_{m}\right)}
\end{aligned}
$$

The scalar field potential $V_{m}(\phi)$ is explained in the next subsection. 


\subsection{Jump conditions for $\phi$}

In the absence of delta function sources for $\phi$, regularity of the solutions requires that $\phi^{\prime}(0)=\phi^{\prime}\left(\rho_{m}\right)=0$. If potentials $V_{0}(\phi) \delta^{(2)}(\rho), V_{m}(\phi) \delta\left(\rho-\rho_{m}\right)$ are included in the Lagrangian, $\phi^{\prime}$ may be nonzero at these points. The effect of $V_{m}$ is easily found by integrating the $\phi$ equation of motion in the vicinity of $\rho_{m}$ assuming $Z_{2}$ symmetry:

$$
\phi^{\prime}\left(\rho_{m}\right)=-\frac{1}{2} \frac{d V_{m}}{d \phi}
$$

The effect of $V_{0}$ is more subtle. One can analyze it by regularizing the $2 \mathrm{D}$ delta function, $\delta^{(2)}(\rho) \rightarrow 1 /\left(\pi L^{\prime}(0) \epsilon^{2}\right)$ for $\rho<\epsilon$, and solving the field equation in the small $\rho$ region using regular boundary conditions, $\phi^{\prime}(0)=0$. The behavior of $\phi$ depends on the choice of potential. For $V_{0}=\lambda\left(\phi-\phi_{0}\right)^{2}$, we find that $\phi \sim c_{1} J_{0}(m \rho)+c_{2} Y_{0}(m \rho)$ in the interior region, with $c_{2} \sim 1 / \ln (\epsilon)$. Therefore in the limit $\epsilon \rightarrow 0$, we recover the boundary condition $\phi^{\prime}(0)=0$ despite the presence of the potential. On the other hand, the linear potential $V_{0}=\lambda \phi$ yields a singular solution, $\phi \sim \phi_{0} \ln (\rho)$ near the origin.

\section{Stabilization in warped model}

Our ultimate goal is to understand the rate of expansion of the universe in models where we live on a codimension-two brane, with the expectation that the Hubble rate is due to the energy density of this 3-brane. Since the present universe has an energy density which is much less than that corresponding to the Planck scale, it is appropriate to treat $\Lambda_{4}$ as a perturbation. We therefore want to first understand the model in the static limit, $\Lambda_{4}=0$. Studies of 5D models have underscored the point that it is essential to stabilize massless volume moduli in order to recover $4 \mathrm{D}$ Einstein gravity at low energies, so we now consider a stabilization mechanism, in this section for the case where the $6 \mathrm{D}$ spacetime is warped by a negative bulk energy density.

\subsection{Stabilization by bulk scalar}

It has been shown [22] that the radial size of the extra dimension is unstable in the AdS soliton model; one way to stabilize it is with a bulk scalar field which is prevented from vanishing by the presence of a potential $V_{m}(\phi)$ on the 4-brane at $\rho_{m}$. Following [20], we make the simple choice $V_{m}(\phi)=-\lambda \phi$, which gives the boundary condition $\phi^{\prime}=\lambda / 2$, as noted in (3.8). In the large $\rho$ region, where $M \sim L \sim e^{2 k \rho / 5}$. In the large and small $\rho$ regions, the solution for $\phi$ is approximately

$$
\begin{array}{ll}
\phi=B_{1} e^{\sigma_{+} \rho}+B_{2} e^{\sigma_{-} \rho}, & \rho \gg 1 / k \\
\phi=\phi_{0}\left(1+m^{2} \rho^{2} / 4+\ldots\right), & \rho \ll 1 / k
\end{array}
$$

with $\sigma_{ \pm}=-k \pm \sqrt{k^{2}+m^{2}}$ and $k=\sqrt{-5 \Lambda_{6} / 8}$. The behavior at $\rho=0$ follows from assuming there is no potential for $\phi$ at $\rho=0$. By matching the two solutions at $\rho \sim 1 / k$, and further assuming that $m \ll k$, one finds that $B_{2}=O\left(m^{4} / k^{4}\right) \phi_{0}, B_{1} \cong \phi_{0}$. Ignoring the $B_{2}$ term, the jump condition at $\rho_{m}$ fixes $\sigma_{+} \phi\left(\rho_{m}\right)=\lambda / 2$. The assumption $m \ll k$ is 
useful for limiting the back reaction of the scalar on the geometry; in the limit $m \rightarrow 0$, $\phi^{\prime} \rightarrow 0$, and the effect of the scalar is to shift the value of $\Lambda_{6}$, so that the solution for the metric would have the same form as that without the scalar. In this regime of parameters, the scalar solution can be approximated as $\phi \cong \phi_{0} e^{m^{2} \rho / 2 k}$.

In solving the jump conditions for the metric, it is useful to consider linear combinations which are independent of either the extra source $\tau^{\prime}$ or the bulk vacuum energy $\Lambda_{6}$. These linear combinations are

$$
\begin{aligned}
& -\lambda \phi+T_{4}=\left(6+\frac{2}{\alpha}\right) \frac{M^{\prime}}{M}+\left(2-\frac{2}{\alpha}\right) \frac{L^{\prime}}{L} \equiv\left(6+\frac{2}{\alpha}\right) \mu+\left(2-\frac{2}{\alpha}\right) \ell \\
& \frac{\alpha \tau^{\prime}}{2 L^{\alpha}}=\frac{L^{\prime}}{L}-\frac{M^{\prime}}{M} \quad \equiv \ell-\mu
\end{aligned}
$$

where all quantities are to be evaluated at $\rho=\rho_{m}$. The right hand side depends only on ratios where the overall normalization of the metric elements cancel out, whereas the left hand side depends on $L$ itself. Using $L=R M^{\prime}, L^{\prime}=R M^{\prime \prime}, L^{\prime}(0)=1-\tau_{3} / 2 \pi$ and (2.2. 2.17) to evaluate $M^{\prime \prime}(0)=2 k^{2} / 5$, we can show that the constant $R$ equals $\left(5 / 2 k^{2}\right)\left(1-\frac{\tau_{3}}{2 \pi}\right)$. (Recall that we have rescaled the $4 \mathrm{D}$ coordinates $x_{\mu}$ so that $M(0)=1$ ). Then $L$ can be expressed as

$$
L=\frac{5}{2} k^{-2}\left(1-\frac{\tau_{3}}{2 \pi}\right) M^{\prime} \cong k^{-1}\left(1-\frac{\tau_{3}}{2 \pi}\right) M
$$

where the latter approximation holds for $\rho \gg 1 / k$. Eqs. (4.2, 4.3) determine $\rho_{m}$, the position of the 4-brane, and they give a constraint on some function of $T_{4}, \tau_{3}$ and $\tau^{\prime}$. The latter is the fine-tuning which must be done in order to obtain a static solution. We will carry this out explicitly below.

\subsection{Back reaction of scalar (or magnetic flux) on metric}

To solve the jump conditions (4.2, 4.3), we need to know how the scalar perturbs the metric. Although we will consider stabilization by the scalar and not the flux, for generality we indicate how the same analysis can be carried out for stabilization by flux. Similarly, we can also compute the perturbation away from the static solution by keeping the effect of the $4 \mathrm{D}$ cosmological constant, even though our immediate goal is to examine the static solution.

Let $\delta \mu$ be the perturbation to $M^{\prime} / M$ in eq. (2.2), which when linearized takes the form

$$
\delta \mu^{\prime}+5 \mu \delta \mu=\frac{1}{8}\left(4 \frac{\Lambda_{4}}{M^{2}}-\phi^{\prime 2}-m^{2} \phi^{2}+\frac{n^{2} \phi^{2}}{L^{2}}+\frac{\beta^{2}}{M^{8}}\right)
$$

The part of $\delta \mu$ in the absence of flux, winding, or cosmological expansion, due only to the stabilization mechanism, is

$$
\begin{aligned}
\delta \mu_{\phi} & \equiv \delta\left(\frac{M^{\prime}}{M}\right)=-\frac{1}{8} M^{-5} \int_{0}^{\rho} d \rho\left(\phi^{\prime 2}+m^{2} \phi^{2}\right) M^{5} \\
& \cong-\frac{m^{2} \phi_{0}^{2}}{64 \cosh ^{2}(k \rho)}\left(\frac{e^{2\left(k+\sigma_{+}\right) \rho}-1}{k+\sigma_{+}}+2 \frac{e^{2 \sigma_{+} \rho}-1}{\sigma_{+}}+\frac{e^{2\left(-k+\sigma_{+}\right) \rho}-1}{-k+\sigma_{+}}\right)
\end{aligned}
$$


It can be shown that in the $m \ll k$ limit in which we are interested, it is always true that $\phi^{2} \ll m^{2} \phi^{2}$, so we dropped the derivative term to obtain (4.11). Similarly the decaying exponential part of $\phi$ gives only subdominant contributions in $m^{2}$, so we approximated $\phi=\phi_{0} e^{\sigma+\rho}$. Now eq. (4.6) can be integrated again to find the deviation in $M$ itself,

$$
\frac{\delta M_{\phi}}{M}=\int_{0}^{\rho} d \rho \delta \mu_{\phi} \cong-\frac{\lambda^{2}}{16 m^{2}} k \rho_{m}\left(\text { at } \rho=\rho_{m}\right)
$$

where in the last approximation we have kept only the leading behavior in $m^{2}$. The last result shows that $\lambda^{2} k \rho_{m} / m^{2}$ must be kept small in order for the perturbation to be under control. Notice that we have used our freedom to impose the boundary condition $\delta M_{\phi}(0)=0$ to preserve the normalization $M(0)=1$.

To compute the analogous shift $\delta \ell_{\phi}$ in $L^{\prime} / L$, we perturb eq. (2.3). In fact it will be convenient to compute $\delta \ell_{\phi}-\delta \mu_{\phi}$,

$$
\delta \ell_{\phi}-\delta \mu_{\phi}=-\left(4+\frac{\ell}{\mu}\right) \delta \mu_{\phi}+\frac{1}{8 \mu}\left(\phi^{2}-m^{2} \phi^{2}\right)
$$

Using $\ell / \mu=\left(5 \operatorname{coth}^{2}(k \rho)-3\right) / 2$ and $\mu=(2 k / 5) \tanh (k \rho)$ for the unperturbed quantities, we can explicitly integrate (4.8) from 0 to $\rho$. The result is

$$
\frac{\delta L_{\phi}}{L}-\frac{\delta M_{\phi}}{M}=\frac{5}{16} \frac{\lambda^{2}}{m^{2}}\left(e^{-2 \sigma_{+} \rho}-\frac{1}{2+m^{2} / k^{2}}+O\left(e^{-2 k \rho}\right)\right)
$$

This result will be essential for finding the size of the radial extra dimension $\rho_{m}$ from the stabilization mechanism.

\subsection{Solution of jump conditions}

We are now ready to solve for $\rho_{m}$ and the fine-tuning between stress-energy components which leads to the static solution. Toward this end, it is useful to rewrite the difference between eqs. (2.2) and (2.1) in the form

$$
\left(M^{4} L(\mu-\ell)\right)^{\prime}=M^{4} L\left(\frac{\Lambda_{4}}{M^{2}}+\frac{n^{2} \phi^{2}}{L^{2}}+\frac{\beta^{2}}{M^{8}}\right)
$$

If we are only interested in stabilization by the Goldberger-Wise mechanism, and the static solution, then the right hand side of (4.10) vanishes, leading to

$$
\ell-\mu=\frac{c}{M^{4} L}=\frac{L^{\prime}(0)}{M^{4} L}
$$

where we evaluated the equation at $\rho=0$ to evaluate the constant of integration $c$. Combining (4.11) with the jump condition (4.3), we find the equation which determines the $\rho_{m}$, namely $2 L^{\prime}(0) / \alpha \tau^{\prime}=M^{4} L^{1-\alpha}$. The only hope for obtaining the desired large hierarchy $\rho_{m} \gg 1 / k$ is in the case where $\alpha=5$. Otherwise we obtain $e^{2(1-\alpha / 5) k \rho_{m}} \sim L^{\prime}(0) / \tau^{\prime}$, which gives a large hierarchy only by tuning $\tau^{\prime}$ to be unnaturally small in units of the $6 \mathrm{D}$ gravity 
scale. On the other hand, in the case $\alpha=5$, we must rely on the small correction (4.9) to find the leading $\rho$ dependence in the jump condition,

$$
y \equiv \frac{2}{5 \tau^{\prime}}\left(1-\frac{\tau_{3}}{2 \pi}\right)=\left(\frac{M}{L}\right)^{4}=1-4\left(\frac{\delta L_{\phi}}{L}-\frac{\delta M_{\phi}}{M}\right)+O\left(e^{-2 k \rho_{m}}\right)
$$

The result for $\rho_{m}$ is

$$
\rho_{m}=-\frac{k}{m^{2}} \ln \left(\frac{4 m^{2}}{5 \lambda^{2}}(1-y)+\frac{1}{2}\right)
$$

This solution only exists if $\tau^{\prime}$ lies in the range

$$
\frac{2\left(1-\frac{\tau_{3}}{2 \pi}\right)}{5\left(1+\frac{5 \lambda^{2}}{8 m^{2}}\right)}<\tau^{\prime}<\frac{2\left(1-\frac{\tau_{3}}{2 \pi}\right)}{5\left(1-\frac{5 \lambda^{2}}{8 m^{2}}\right)}
$$

This range could have had a large upper limit if $5 \lambda^{2} / 8 m^{2} \lesssim 1$. However we found in (4.7) that in fact we must have $\lambda^{2} / m^{2}<1 /\left(k \rho_{m}\right)$ in order for perturbation theory to not break down. So we see that there is still a fine tuning on $\tau^{\prime}$ for this model to give a large hierarchy. It is qualitatively different from the kind of tuning that would occur in models with $\alpha<5$; for these one would need to make the magnitude of $\tau^{\prime}$ exponentially smaller than its natural scale in terms of the $6 \mathrm{D}$ gravity scale. For $\alpha=5$, the magnitude of $\tau^{\prime}$ can be natural, but it must be very close to a specific value. One virtue of the scalar field is that it reduces the amount of tuning which is necessary. A similar tuning was present in the absence of the scalar field: 4.12 shows that $y$ must be exponentially close to 1 if $\phi$ is absent, and if we insist on getting a large hierarchy. Once $\phi$ is introduced, $y$ must still be close to 1 , but no longer with exponential precision.

We could simply give up the tuning of $\tau^{\prime}$ and live with a model which does not try to solve the hierarchy problem. But since the main purpose of this paper is to explore the strange cosmological properties of this kind of model, questions of naturalness of the choice of parameters are secondary. It is convenient to adhere to the strongly warped case, since the hierarchy simplifies the algebraic analysis. We have seen that even in the completely unwarped case, similar behavior is revealed, concerning the unusual dependence of the Hubble rate on the brane tension is concerned.

The remaining jump condition (4.2) is the fine tuning that must be imposed to make the effective $4 \mathrm{D}$ cosmological contant $\Lambda_{4}$ vanish:

$$
T_{4}-\frac{\lambda^{2} k^{2}}{2 m^{2}}-\frac{16}{5} k=5 \tau^{\prime} e^{-2 k \rho_{m}}\left(1+\frac{5 \lambda^{2}}{16 m^{2}} k \rho_{m}\right)
$$

underscoring the fact that ultimately one needs an additional mechanism to fully solve the cosmological constant problem in these models. Our purpose here is not to address the problem of the 4-D cosmological constant, but rather the possible cosmological solutions of codimension-2 braneworlds.

\subsection{Stabilization by magnetic flux?}

We can attempt to stabilize the warped solution using magnetic flux; however since the effects of the flux are highly suppressed at large $\rho$ because of the large exponential factor 
$1 / M^{8}$, it is not clear that it actually can stabilize the highly warped solution. Following the same steps as for the scalar field, we find

$$
\delta \mu_{\beta}=\frac{\beta^{2}}{8 \cosh ^{2}(k \rho)} \int_{0}^{\rho} d \rho M^{-3} \cong \frac{\beta^{2}}{8 k \cosh ^{2}(k \rho)}\left(c_{0}+c_{1} e^{-6 k \rho / 5}+c_{2} e^{-c_{3} k \rho}\right)
$$

where the approximate fit has $c_{0}=1.38, c_{1}=-1.91, c_{2}=-c_{0}-c_{1}, c_{3}=-\left(1+\frac{6}{5} c_{1}\right) / c_{2}$ and in the large $\rho$ limit

$$
\frac{\delta L_{\beta}}{L}-\frac{\delta M_{\beta}}{M} \cong \frac{5 \beta^{2}}{16 k^{2}}\left(d_{0}+d_{1} e^{-2 k \rho}\right)
$$

where $d_{0}=-0.66, d_{1}=5.5$. The resulting equation for $\rho_{m}$ has the same form as (4.12), except now the term $\delta L_{\beta} / L-\delta M_{\beta} / M$ depends on $\rho_{m}$ only through $e^{-2 k \rho_{m}}$, which is of the same order as terms we ignored in (4.12). As explained there, this kind of dependence does not allow for a large hierarchy unless $\tau^{\prime}$ is tuned with exponential precision to a special value. This is exaclty the same situation as in the model with no stabilization, leading one to suspect that magnetic flux does not actually stabilize this model, unless the warping is weak (which would be the case if $\Lambda_{6}>0$ ).

\section{Friedmann equation in warped model}

We now come to the main point of the paper, in the context of the AdS-type models: the rate of expansion in the models we have described depends on the energy density (tension) of the standard model 3-brane in a strange way, relative to our expectations from 4D general relativity. Such an outcome would be less surprising if the low energy limit of the model was a scalar-tensor theory of gravity, in which the coupling of the extra massless scalar to the brane tension could explain the exotic effect. However we will note that the conclusion is valid even when moduli have been stabilized. We now demonstrate this in the warped case of the expanding AdS soliton model, (2.18), supplemented by a scalar field which stabilizes the radion by the Goldberger-Wise mechanism [36, 17, 20, 22].

The main work has already been done in the previous section; there we found the back reaction to the metric by treating the scalar field as a perturbation. In the same way we can find the perturbation to the static solution due to a small rate of expansion by treating the $\Lambda_{4} / M^{2}$ term to first order. We have a double expansion, in $\Lambda_{4}$ and in $\phi^{2}$. We will work to first order in either quantity, but not including the mixed terms of order $\Lambda_{4} \phi^{2}$.

The perturbation $\delta \mu_{\Lambda}$ analogous to (4.6) is

$$
\begin{aligned}
\delta \mu_{\Lambda} & =\frac{1}{2} M^{-5} \int_{0}^{\rho} d \rho \frac{\Lambda_{4}}{M^{2}} M^{5} \\
& \cong \frac{5 \Lambda_{4}}{12 \cdot 2^{6 / 5} k} \cosh ^{-2}(k \rho)\left(e^{6 k \rho / 5}-1-a\left(e^{-k b \rho}-1\right)\right)
\end{aligned}
$$

where $a=\left(2^{6 / 5}-1\right)^{2}, b=(6 / 5)\left(2^{6 / 5}-1\right)^{-1}$. The integral cannot be done analytically, but the appproximation (5.2) is chosen to give the correct large $\rho$ behavior, and it matches the exact value and its first derivative at $\rho=0$; it is accurate to $0.6 \%$ everywhere, as shown in fig. 3. 


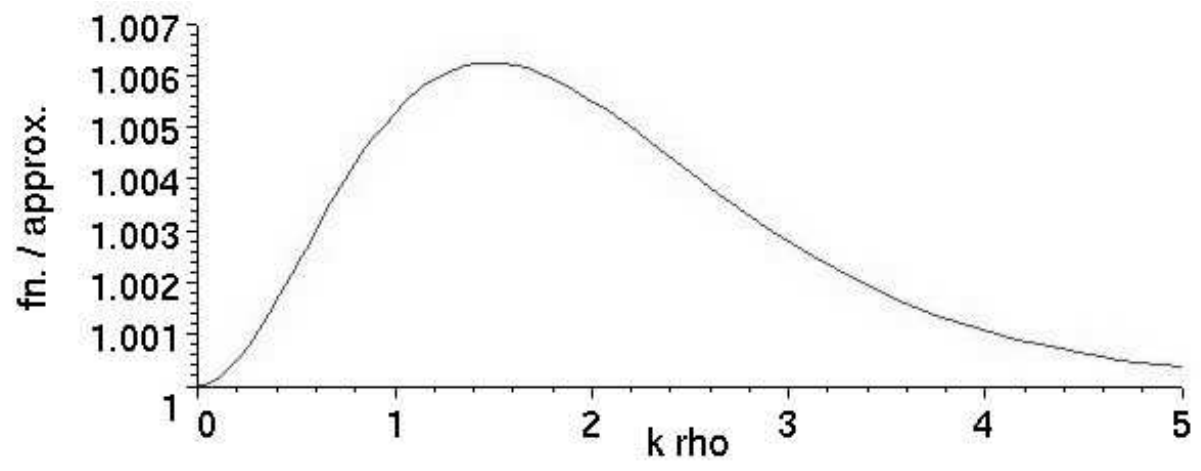

Figure 3: Ratio of the exact value of the integral in (4.11) to the approximation (5.2) as a function of $k \rho$.

The shift in $\delta \ell$ is given by

$$
\delta \ell_{\Lambda}-\delta \mu_{\Lambda}=-\left(4+\frac{\ell}{\mu}\right) \delta \mu_{\Lambda}+\frac{\Lambda_{4}}{2 \mu M^{2}}
$$

By numerical evaluation and empirically fitting, we find that the following expression is a very good approximation to the integral of (5.3):

$$
\frac{\delta L_{\Lambda}}{L}-\frac{\delta M_{\Lambda}}{M} \cong \frac{5}{4} \frac{\Lambda_{4}}{k^{2}}\left(-1+\cosh ^{-\frac{4}{5}}(k \rho)\right)
$$

This captures with great accuracy asymptotic approach of the solution to its large $\rho$ limit, as well as having the correct small $\rho$ behavior. The comparison between the exact result and the approximation is shown in fig. 4.

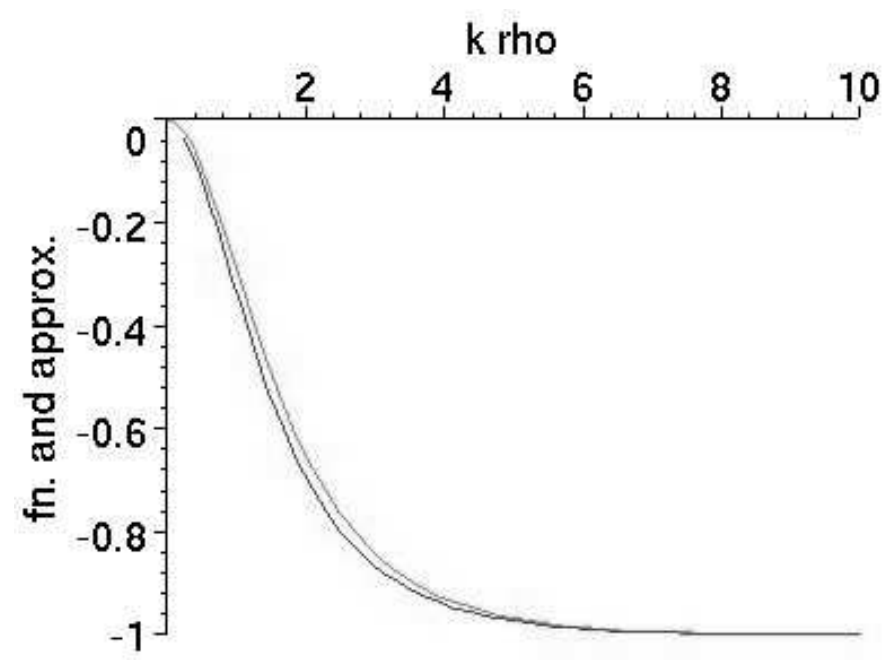

Figure 4: Numerical integration of (5.3) versus $k \rho$, and the approximation (5.4). 
The new contribution to the jump condition determining the combination $\ell-\mu$ from (4.10) gives

$$
\begin{aligned}
\ell-\mu & =\frac{1}{M^{4} L}\left(L^{\prime}(0)-\int_{0}^{\rho} d \rho \frac{\Lambda_{4}}{M^{2}} M^{4} L\right) \\
& =\frac{L^{\prime}(0)}{M^{4} L}\left(1-\frac{5 \Lambda_{4}}{6 k^{2}}\left(M^{3}-1\right)\right)
\end{aligned}
$$

where we used (4.4) to perform the integral exactly. This modifies (4.12) by giving a new term proportional to $\Lambda_{4}$ :

$$
y \equiv \frac{2}{5 \tau^{\prime}}\left(1-\frac{\tau_{3}}{2 \pi}\right) \cong 1+\frac{5 \lambda^{2}}{16}\left(e^{-2 \sigma_{+} \rho}-\frac{1}{2}\right)+\frac{5 \Lambda_{4}}{6 k^{2}} M^{3}
$$

where we have ignored all exponentially small corrections.

Let us now recall what is the origin of the expansion of the universe according to our assumptions. We are interested in starting from a static configuration, and then perturbing it by adding some energy density on the 3-brane. Of course it is possible that the expansion of our own universe could be due to a small mismatch of bulk energy densities which are distributed in the extra dimensions. But our specific interest in this paper is to see how the energy density on the 3-brane affects the Hubble expansion. Therefore we will make the assumption that the quantity whose value is relaxed relative to the static fine-tuned situation is $\tau_{3}$; hence in our perturbation series $\delta \tau_{3}$ and $\Lambda_{4}$ are of the same order. When comparing (5.6) to the corresponding relation (4.12) for the static solution, there is one other quantity which must in general vary: the position of the 4 -brane $\rho_{m}$ will deviate by some amount $\delta \rho_{m}$. However, all terms containing $\delta \rho_{m}$ are or order $\Lambda_{4} \phi^{2}$ or $\Lambda_{4}^{2}$ and so can be neglected. Therefore the difference between $(\sqrt[5.6]{ })$ and (4.12) gives

$$
\Lambda_{4}=-\frac{3 k^{2}}{5 \pi} \frac{\delta \tau_{3}}{1-\frac{\tau_{3}}{2 \pi}} \cosh ^{-\frac{6}{5}}\left(k \rho_{m}\right)
$$

where we used the constraint (4.14) to eliminate $\tau^{\prime}$. This is the result we have been seeking, which shows an unexpected relation between $\Lambda_{4}$ and $\delta \tau_{3}$ : a decrease in the energy density on the 3-brane leads to cosmological expansion in this model.

It is straightforward to give the more general version of eq. (5.7), for arbitrary values of $\alpha$, and without assuming that we are perturbing around a static solution. We find that

$$
\Lambda_{4}=\frac{6 k^{2}}{5\left(M^{3}-1\right)}\left(1-\frac{\alpha M^{4} L^{1-\alpha} \tau^{\prime}}{2\left(1-\frac{\tau_{3}}{2 \pi}\right)}\right)
$$

where $M$ and $L$ are to be evaluated at $\rho_{m}$. This shows that the surprising dependence on $\tau_{3}$ is not due to fine tuning of parameters. The Hubble rate is a decreasing function of $\tau_{3}$ quite generally in this model. By choosing $\tau_{3}$ so as to make $\Lambda_{4}=0$, and perturbing $\tau_{3} \rightarrow \tau_{3}+\delta \tau_{3}$, one recovers eq. (5.7).

When we perturb the other jump condition (4.2), we obtain an equation for the shift in $\rho_{m}$,

$$
\left(\mu^{\prime}+\frac{1}{5}\left(\ell^{\prime}-\mu^{\prime}\right)\right) \delta \rho_{m}+\delta \mu_{\Lambda}+\frac{1}{5}\left(\delta \ell_{\Lambda}-\delta \mu_{\Lambda}\right)=0
$$


which gives

$$
\delta \rho_{m}=\frac{-5 \Lambda_{4}}{32 k^{3}} \frac{e^{2 k \rho_{m}}}{\cosh ^{\frac{4}{5}}\left(k \rho_{m}\right)}=\frac{3}{20 \pi} \frac{\delta \tau_{3}}{k \tau^{\prime}}
$$

\section{Stability of unwarped model}

As already pointed out in eq. (2.20), it is possible to find unwarped solutions [13, 14] where the bulk is a two-sphere stabilized by magnetic flux. In these models the expansion rate is insensitive to the three brane tension. To be sure that this unusual behavior is not related to the presence of unstabilized moduli, in this section we perform a more complete analysis of the stability of the model than was done in ref. [30]. In the following we will set $M=1$. We will consider in detail the static case and then give the main results of the analysis in the presence of the expansion.

In the static case $\Lambda_{4}=0$ and, from eqs. (2.1)-(2.2) we have

$$
\ell^{2}+\ell^{\prime}=-\beta^{2}, \quad \Lambda_{6}=\frac{\beta^{2}}{2} .
$$

The fluctuations of this model may be classified according to four-dimensional Lorentz transformations. On top of the usual transverse and traceless tensor (i.e. $\partial_{\mu} h_{\nu}^{\mu}=h_{\mu}^{\mu}=0$ ) corresponding to five degrees of freedom there are three divergenceless vector modes (corresponding to nine degrees of freedom) and seven scalar modes. Overall, the perturbed six-dimensional metric has 21 degrees of freedom. These 21 fluctuations of different spin transform under coordinate transformations parametrized by the infinitesimal shift $\epsilon_{A} \equiv$ $\left(\epsilon_{\mu}, \epsilon_{\rho}, \epsilon_{\theta}\right)$. The infinitesimal shift $\epsilon_{\mu}$ along the four-dimensional spacetime can be decomposed, in turn, as the derivative of a scalar and a divergenceless vector, i.e. $\epsilon_{\mu}=\partial_{\mu} \epsilon+\zeta_{\mu}$. It is now easy to see that there are 3 scalar gauge functions $\left(\epsilon_{\rho}, \epsilon_{\theta}\right.$ and $\left.\epsilon\right)$ and one vector gauge-function. Of the seven scalar degrees of freedom, three of them can be gauged away by using the scalar gauge functions. Alternatively, the seven gauge-dependent scalars can be rearranged into four gauge-invariant scalar fluctuations. In the context of the study of six-dimensional Abelian vortices [40, 41] it was shown that a convenient gauge choice brings the line element in the form

$$
d s^{2}=(1-2 \psi) \eta_{\mu \nu} d x^{\mu} d x^{\nu}+(1+2 \xi) d \rho^{2}+L(\rho)^{2}(1-12 \varphi) d \theta^{2}+2 \pi L(\rho) d \rho d \theta
$$

where $\eta_{\mu \nu}$ is the Minkowski metric. Since all the scalar gauge functions are completely fixed, no spurious gauge modes will appear. Furthermore, it can be shown that the scalar fluctuations appearing in (6.2) obey the same evolution equations of the four gauge-invariant fluctuations which have been defined, in general terms, in 41.

The four scalar fluctuations of the geometry are coupled, through the perturbed Einstein equations

$$
\delta R_{A B}=\delta \tau_{A B}, \quad \tau_{A B}=T_{A B}-\frac{1}{4} T_{C}^{C} G_{A B}
$$

to the scalar fluctuations of the sources whose evolution can be obtained by perturbing to first order

$$
\partial_{A}\left(\sqrt{|G|} F^{A B}\right)=0
$$


The two relevant fluctuations of the source are the fluctuations of the $\rho$ and $\theta$ components of the vector potential. These fluctuations will be donoted as $A_{\rho}$ and $A_{\theta}$. The divergencefull part of the four-dimensional vector potential is also a scalar but it can be gauged away by using the $U(1)$ gauge symmetry.

Now that the gauge is fully fixed, we are ready to find the spectrum of fluctuations for the class of unwarped models defined by (6.1). In connection with the automatic adjustment of the solutions to cancel out any dependence on the tension of the 3-branes, we are specifically interested in the radial modes and, therefore, the possible dependence of the perturbed quantities upon the coordinate $\theta$ will be ignored. Using (6.2) the various components of eq. (6.3) are

$$
\begin{aligned}
\mu \neq \nu: & \xi=\varphi+2 \psi, \\
\mu=\nu: & \psi^{\prime \prime}+\ell \psi^{\prime}+\square \psi=-\frac{\beta}{2 L} A_{\theta}^{\prime}+\frac{\beta^{2}}{2}(\xi-\varphi), \\
\rho \rho: & \varphi^{\prime \prime}+4 \psi^{\prime \prime}+\ell\left(2 \varphi^{\prime}+\xi^{\prime}\right)-\frac{3}{2} \beta^{2} \varphi-\Lambda_{6} \xi-\square \xi=\frac{3}{2} \frac{\beta}{L} A_{\theta}^{\prime} \\
\theta \theta: & \varphi^{\prime \prime}+\ell\left(2 \varphi^{\prime}+\xi^{\prime}+4 \psi^{\prime}\right)-\frac{3}{2} \beta^{2} \varphi+\square \varphi+2\left[\ell^{2}+\ell^{\prime}+\frac{3}{4} \beta^{2}\right] \xi=\frac{3}{2} \frac{\beta}{L} A_{\theta}^{\prime}, \\
\mu \rho: & \varphi^{\prime}+\ell(\varphi+\xi)+3 \psi^{\prime}=\frac{\beta}{L} A_{\theta}, \\
\mu \theta: & \pi^{\prime}+2 \ell \pi=-\frac{\beta}{L} A_{\rho}, \\
\theta \rho: & \square \pi=0 .
\end{aligned}
$$

where $\square$ denotes the four-dimensional D'Alembertian, $-\partial_{t}^{2}+\partial_{\vec{x}}^{2}$. Eqs. (6.5)-6.11) should be supplemented by the evolution equations describing the fluctuations of the sources which can be obtained by perturbing eq. (6.4) to first order:

$$
\begin{aligned}
& A_{\theta}^{\prime \prime}-\ell A_{\theta}^{\prime}+\beta L\left(4 \psi^{\prime}+\xi^{\prime}-\varphi^{\prime}\right)+\square A_{\theta}=0, \\
& \square A_{\rho}=0, \quad A_{\rho}^{\prime}=0 .
\end{aligned}
$$

Notice that $\pi$ and $A_{\rho}$ are decoupled from the other equations. The resulting system of coupled equations (6.11) and (6.13) implies that both $\pi$ and $A_{\rho}$ are massless excitations. Furthermore, since $A_{\rho}$ is constant, eq. (6.10) can be easily solved with the result that

$$
\pi(\rho)=\frac{c_{\pi}}{L^{2}}-\beta A_{\rho} \int d \rho L(\rho) .
$$

However this mode is not normalizable, hence it is unphysical. In the action perturbed to second order the kinetic term for $\pi$ appears multiplied by only one factor of $L$. Thus the kinetic term for $\pi$ diverges as $\rho \rightarrow 0$, in agreement with the findings of [41].

The remaining longitudinal degrees of freedom (i.e. $\varphi, \psi, \xi$ and $A_{\theta}$ ) satisfy a coupled set of linear differential equations whose eigenvalues determine the stability of the solution (6.1). The boundary conditions to be imposed at the origin are [22]:

$$
\varphi(0)+\xi(0)=0, \quad \psi^{\prime}(0)=0, \quad A_{\theta}^{\prime}(0)=0 .
$$


If we now consider eq. (6.5) we see that, for $\rho \rightarrow 0$, eqs. (6.15) imply

$$
\phi(0)+\psi(0)=0
$$

It is convenient to define two new variables:

$$
\psi=\frac{X-Y}{2}, \quad \xi=\frac{X+Y}{2} .
$$

With these variables we will have that the linear combination $3 \times(\mu, \nu)+(\theta, \theta)$ leads to

$$
X^{\prime \prime}+3 \ell X^{\prime}+\left(\square-\beta^{2}\right) X=\beta^{2} Y
$$

while the difference of the $(\rho, \rho)$ and $(\theta, \theta)$ components of the perturbed Einstein equations (6.7) and (6.8) gives

$$
X^{\prime \prime}-Y^{\prime \prime}-\ell\left(X^{\prime}-Y^{\prime}\right)=\square Y
$$

and eq. (6.9) determines the gauge field

$$
A_{\theta}=\frac{L}{\beta}\left(X^{\prime}+2 \ell Y\right) .
$$

In terms of $X$ and $Y$ the boundary conditions (6.15) are now $X^{\prime}(0)=Y^{\prime}(0)$ and $Y(0)=0$.

It is easy to verify that one solution to the coupled equations $(6.18,6.19)$ matches the results of ref. [30], with $X=$ constant, $Y=0$, and mass eigenvalue

$$
m_{r}^{2}=\beta^{2}
$$

which is the radion mass squared of this model. Although the analysis of [30] was in a $4 \mathrm{D}$ effective theory, their result is exact since the solution has no dependence on the extra dimension. The constancy of the wave function shows that this must indeed be the ground state of the system (6.18, 6.19), since nonconstant solutions should have higher energy and correspond to Kaluza-Klein excitations of the ground state. Nevertheless to be thorough we have conducted a numerical search for other eigenstates using the shooting method, and verified that (6.21) is indeed the ground state solution.

The same analysis can be done in the case where the background solution has de Sitter expansion, as in eq. (2.20), rather than being static. The system (6.18, 6.19) becomes

$$
\begin{aligned}
& X^{\prime \prime}+3 \ell X^{\prime}+X\left(\square-\beta^{2}+\frac{33}{4} H^{2}\right)=\left(\beta^{2}+12 H^{2}\right) Y \\
& X^{\prime \prime}-Y^{\prime \prime}-\ell\left(X^{\prime}-Y^{\prime}\right)=\left(\square+\frac{9}{4} H^{2}\right)
\end{aligned}
$$

The form of the bulk solution is the same as when $H=0$, but the mass eigenvalue generalizes to

$$
m_{r}^{2}=\beta^{2}-\frac{33}{4} H^{2}
$$

showing that a sufficiently large rate of expansion destabilizes the compactification. 
Let us now discuss the vector modes, which must be considered in a fully consistent decomposition of the metric and source fluctuations $6 \mathrm{D}$. It was recently shown in 14 that a massless graviphoton field is present in the low-energy spectrum. In the following we restrict ourselves to the spectrum of the vector excitations with only radial dependence. The $\theta$ dependence of the perturbed quantities, considered in [40], would presumably correspond to KK excitations of the lowest modes which we seek here.

Denoting by $V_{\mu}$ and $Z_{\mu}$ the divergenceless graviphoton fields appearing, respectively, in the $(\mu, \rho)$ and $(\mu, \theta)$ components of the perturbed metric, the equations for the coupled system of vector fluctuations reads:

$$
\begin{aligned}
\mu \neq \nu: & V_{\mu}^{\prime}+\ell V_{\mu}=0, \\
\mu \rho: & \square V_{\mu}=0, \\
\mu \theta: & Z_{\mu}^{\prime \prime}+\ell Z_{\mu}^{\prime}+\ell^{\prime} Z_{\mu}+\square Z_{\mu}+2 \beta A_{\mu}^{\prime}=0 \\
\mu: & A_{\mu}^{\prime \prime}+\ell A_{\mu}^{\prime}+\square A_{\mu}-\beta\left[Z_{\mu}^{\prime}+\ell Z_{\mu}\right]=0,
\end{aligned}
$$

where $A_{\mu}$ is the divergenceless fluctuation of the vector potential and eq. (6.28) follows from the perturbed component of the evolution equation of the gauge field.

From eq. (6.26) it follows that $V_{\mu}$ is always massless in perturbation theory, as was argued in [14]. This conclusion, valid for radial excitations, follows from the cancellation of the contribution of $\Lambda_{6}$ and the magnetic flux in the perturbed equations. However eq. (6.25) implies that $V_{\mu} \sim L^{-1}$. Like the $\pi$ mode in (6.14) this mode is not normalizable and therefore unphysical.

To analyze the coupled system of excitations for $Z_{\mu}$ and $A_{\mu}$ notice that if the mode is massless, then eq. (6.28) can be integrated once to find that $A_{\mu}^{\prime}=\beta Z_{\mu}$. This allows us to rewrite the equation for $Z_{\mu}$ in a decoupled form:

$$
Z_{\mu}^{\prime \prime}+\ell Z_{\mu}^{\prime}+\ell^{\prime} Z_{\mu}+2 \beta^{2} Z_{\mu}=0
$$

It is already known that in the case of the six-dimensional Abelian vortex there exists a normalizable vector zero mode [40, 41] (see also [42]). In our case, recalling (6.1), the zero mode takes the form

$$
A_{\mu} \sim \beta \int^{\rho} L d \rho^{\prime}, \quad Z_{\mu} \sim L
$$

which is the ground state solution of eqs. 6.27) and (6.28). This mode is normalizable since the canonical fields related to $A_{\mu}$ and $Z_{\mu}$ are simply the original ones multiplied by $\sqrt{L}$. The localization of the gauge mode related to $A_{\mu}$ was recently invoked [40, 41, 42] as a mechanism in order to localize gauge fields in the presence of thick Abelian strings in six-dimensions.

The masslessness of the graviphoton mode is not a concern, as would be a massless scalar mode, due to the fact that the graviphoton couples only to $T_{\mu \theta}$, which vanishes on the 3-brane. Since the graviphoton does not couple to standard model matter, it is not phenomenologically constrained like a Brans-Dicke scalar. Moreover it does not seem a likely candidate for explaining the tuning of the bulk geometry in response to a 3-brane tension, since this is a deformation which looks like a purely scalar mode. 


\section{Discussion and conclusions}

We have made a comprehensive review of $6 \mathrm{D}$ cosmologies which contain at least one 3-brane where the standard model could presumably be localized. Emphasis was given to several solutions in which the rate of expansion of the universe is either insensitive to the tension of a 3-brane, eqs. (2.14, 2.19), or else depends on $\tau_{3}$ in a way which is at odds with $4 \mathrm{D}$ general relativity eq. (5.7). This is despite the fact that we are considering values of $\tau_{3}$ which are well below the scale of compactification and the mass scale of moduli which could account for a departure from conventional gravity. If the theories under consideration contained a massless radion, for example, one would not be so surprised to see such behavior. However both of the models we have focused on do have stabilized moduli. The AdS soliton model has only a single radion among the fluctuations with azimuthal symmetry. It is massless for $\alpha=5$ and has an exponentially small negative (mass) ${ }^{2}$ for $\alpha<5$ in the absence of stabilization, but with the Goldberger-Wise mechanism it acquires a positive (mass) ${ }^{2}$ of order $m^{4} k^{-2} \phi^{2}\left(\rho_{m}\right) e^{-2 k \rho_{m} / 5} \sim \lambda^{2} e^{-2 k \rho_{m} / 5}$ [22]. For realistic values of the hierarchy, this gives a radion mass of order $\mathrm{MeV}$, which is safe because the couplings of the radion to the 3-brane are suppressed by the $4 \mathrm{D}$ Planck scale. And in the previous section we have confirmed the stability of the unwarped "football-shaped" model by doing an exhaustive study of its perturbation spectrum.

The tensions of 3-branes in two 6D theories fail to contribute to the Hubble rate in the expected way at low energies, despite the fact that these theories have no additional massless scalars that might explain a deviation from 4D general relativity. Instead, the explanation of this apparent failure of decoupling lies in the special way in which a $2 \mathrm{D}$ manifold's internal geometry responds to the tension of a codimension two brane. This can be seen from a direct dimensional reduction of the 6D theory to four dimensions [9].

Consider the metric (1.1) with a fluctuating $4 \mathrm{D}$ metric, $g_{4, \mu \nu}\left(x_{\mu}\right)$. When we integrate the $6 \mathrm{D}$ Einstein-Hilbert action over the coordinates $y_{i}$ of the internal space, we obtain

$$
\begin{aligned}
\mathcal{L}_{4, \mathrm{eff}} & =\frac{1}{2 \kappa_{6}^{2}} \sqrt{\left|g_{4}\right|} \int d^{2} y \sqrt{g_{2}} M^{2} R_{4}-V_{4, \mathrm{eff}} \\
V_{4, \mathrm{eff}} & =\int d^{2} y M^{4}\left(\sqrt{g_{2}}\left(-\frac{1}{2 \kappa_{6}^{2}} R_{2}+\Lambda_{6}+\frac{1}{4} F^{2}+\ldots\right)+\sum_{i} \tau_{3, i} \delta^{(2)}\left(y-y_{i}\right)\right)
\end{aligned}
$$

where $R_{n}$ is the $n$-dimensional curvature constructed from $g_{n, \mu \nu} ; \tau_{3, i}$ is the tension of the $i$ th 3-brane, and ... denotes contributions from other sources such as the bulk scalar field or a 4-brane. In the unwarped solution, where $\tau_{3,1}=\tau_{3,2} \equiv \tau_{3}$, there are two cancellations which make $R_{4}$ independent of $\tau_{3}$. First, the curvature $R_{2}$ has singular contributions that exactly cancel the delta function terms explictly involving the tensions. One can see the crucial difference between codimension one and two branes by examining their respective contributions to $R_{2}$ [34]:

$$
\frac{R_{2}}{\kappa_{6}^{2}}=2 \tau_{3} \delta^{(2)}(y)+\frac{1}{2} T_{4} \delta^{(1)}\left(y-y_{4}\right)+\text { nonsingular terms }
$$

The coefficient of the $2 D$ delta function is such as to cancel the similar contribution to the action from the 3-brane's stress-energy, whereas this cancellation is not exact for the 
4-brane. The second cancellation of the dependence on the 3-brane tension is this: $\tau_{3}$ enters implicitly through the volume of the extra dimension, $\sqrt{g_{2}(0)}=L^{\prime}(0)=1-\tau_{3} / 2 \pi$ which multiplies the entire nonsingular part of the action, including the coefficient of $R_{4}$ in (7.1), which gives the 4D Planck mass:

$$
M_{p}^{2}=\frac{1}{\kappa_{6}^{2}} \int d^{2} y \sqrt{g_{2}} M^{2}(y)
$$

These two factors of $L^{\prime}(0)$ cancel out of the Friedmann equation, $H^{2}=V_{4, \text { eff }} / 3 M_{p}^{2}$, so that the Hubble rate is determined completely by bulk quantities, independent of the brane tension. Notice that one reason we can make this argument so simply in the unwarped case is the fact that the bulk solution changes only through a rescaling of $L$ as a result of changing the brane tension.

When we try to make a similar argument to deduce the form of the Friedmann equation in the warped solution, it is not straightforward, because $M$ and $L d o$ change from their static forms when the brane tension is changed, which induces a nonzero value of $\Lambda_{4}$. It is still true that the delta function term $\tau_{3} \delta^{(2)}(y)$ is cancelled by a singular term of exactly the same magnitude in $\sqrt{g_{2}} R_{2}$. But now there are additional terms of order $\Lambda_{4}$ from the perturbation to the bulk part of the solution. These did not appear in the unwarped case. The essential difference between the two models is that the jump conditions at the 4-brane bring in a new source of dependence on $L$, hence the 3-brane tension, which does not exist in the type 1 solution or in the unwarped type 3 solution. These observations were also made in [9].

The hope would be to solve the cosmological constant problem by using bulk supersymmetry to ensure $\Lambda_{4}=0$, in a model where $\Lambda_{4}$ is independent of the 3 -brane tension. Then $\Lambda_{4}$ will be insensitive to all quantum corrections to the vacuum energy which arise if the standard model is confined to the 3-brane. However to describe realistic cosmology, one needs to depart from pure de Sitter space and look for Friedmann-Robertson-Walker solutions. In fact, it is not obvious that solutions to the 6D Einstein equations exist when cosmological matter and radiation are added to the brane. Let us suppose the line element has the rather general form

$$
d s^{2}=-N^{2} d t^{2}+M^{2} d \vec{x}^{2}+B^{2} d \rho^{2}+L^{2} d \theta^{2}
$$

where all the metric functions can depend on $t$ as well as $\rho$, and consider the terms in the Einstein equations which can have delta function singularities:

$$
\begin{aligned}
& 00: 3\left(\frac{M^{\prime \prime}}{M}+\frac{M^{\prime} L^{\prime}}{M L}\right)+\frac{L^{\prime \prime}}{L} \quad \sim \epsilon B^{2} \delta^{(2)}(\rho) \\
& \text { ii : } \quad 2\left(\frac{M^{\prime \prime}}{M}+\frac{M^{\prime} L^{\prime}}{M L}\right)+\left(\frac{N^{\prime \prime}}{N}+\frac{N^{\prime} L^{\prime}}{N L}\right)+\frac{L^{\prime \prime}}{L} \sim p B^{2} \delta^{(2)}(\rho) \\
& \theta \theta: \quad 3\left(\frac{M^{\prime \prime}}{M}+\frac{M^{\prime} L^{\prime}}{M L}\right)+\left(\frac{N^{\prime \prime}}{N}+\frac{N^{\prime} L^{\prime}}{N L}\right) \quad \sim 0
\end{aligned}
$$

In all the solutions we have considered, the energy density of the brane is given by $\varepsilon=$ $-p=\tau_{3}$, and $M^{\prime}=N^{\prime}=0$ at $\rho=0$, so that there is no singular part in $M^{\prime \prime}$ or $N^{\prime \prime}$. 
To obtain $\varepsilon \neq-p$, it is necessary to have singular behavior not only in $L^{\prime \prime} / L$, but also in the other terms. However $L^{\prime \prime} / L$ gives a 2D delta function $\delta^{(2)}(\rho) \sim \delta(\rho) / \rho$ by virtue of $L$ vanishing like $\rho$. We can only get this kind of behavior from $M^{\prime \prime} / M$ if $M$ also vanishes, which is not physically sensible since the standard model requires a nonvanishing metric.

The only way we see to admit a general equation of state is to give the 3 -brane a finite radius $\rho_{0}$. A solution to the equation $M^{\prime \prime}+M^{\prime} / r=-\varepsilon M \delta^{(2)}(\rho)$ can be found if $\delta^{(2)}(\rho)$ is a regularized delta function:

$$
M(\rho)= \begin{cases}J_{0}(a \rho), & \rho<\rho_{0} \\ J_{0}\left(a \rho_{0}\right)+a \rho_{0} J_{1}\left(a \rho_{0}\right) \ln \frac{\rho_{0}}{\rho}, & \rho>\rho_{0}\end{cases}
$$

where $a=\sqrt{\varepsilon /\left(\pi \rho_{0}^{2}\right)}$. For branes of codimension one, the interesting corrections to the Hubble rate come from terms like $\left(M^{\prime} / M\right)^{2}$, which are nonsingular and have an unambiguous value proportional to $\varepsilon^{2}$. For the regularized codimension two brane, $\left(M^{\prime} / M\right)^{2} \sim$ $\left(\varepsilon \rho / \rho_{0}^{2}\right)^{2}$ which goes like $1 / \rho_{0}^{2}$ when averaged over the brane. Thus we expect to get results which are sensitive to the internal structure of the brane, unlike the clean predictions that came from the codimension one case. Of course, unambiguous predictions could be made in a specific theory, for example where the brane was a cosmic string defect coming from an Abelian Higgs model. Whether the interesting cosmological properties of codimension two branes will survive after regularizing them requires further investigation.

Acknowledgment. We thank Daniel Chung, Rob Leigh and Dominik Schwarz for valuable discussions. J.C., J.D. and J.V. are supported in part by Canada's National Sciences and Engineering Research Council.

\section{Appendix}

\section{A. Perturbation to AdS soliton from small $\Lambda_{4}$}

Let us write $E=E_{0}+\delta E$, where $E_{0}=a z_{0}^{2}$ and $\delta E=-b z_{0}^{6 / 5}$ is an energy shift relative to the static solution which allows us to keep the same starting value $z_{0}$; then $(E-U)$ can be written as

$$
E-U(z)=a\left(z_{0}^{2}-z^{2}\right)+b\left(z^{6 / 5}-z_{0}^{6 / 5}\right)
$$

and the $b$ term can be consistently treated as a perturbation, even in the region $\rho \cong 0$, $z \cong z_{0}$. The integral (2.11) gives

$$
\begin{aligned}
\rho & =k^{-1} \ln \left(\frac{z}{z_{0}}+\sqrt{\left(\frac{z}{z_{0}}\right)^{2}-1}\right)-\delta \rho(z) ; \\
\delta \rho(z) & =\frac{b}{k^{3}} \int_{z_{0}}^{z} \frac{z^{6 / 5}-z_{0}^{6 / 5}}{\left(z^{2}-z_{0}^{2}\right)^{3 / 2}} d z+O\left(b^{2}\right) \\
& \cong \frac{b z_{0}^{4 / 5}}{k^{3}}\left\{\begin{array}{l}
1.368+\frac{5}{4}\left(\frac{z_{0}}{z}\right)^{4 / 5}-\frac{1}{2}\left(\frac{z_{0}}{z}\right)^{2}+O\left(\left(\frac{z_{0}}{z}\right)^{14 / 5}\right), z \gg z_{0} \\
0.85 \epsilon^{1 / 2}-0.18 \epsilon^{3 / 2}+0.062 \epsilon^{5 / 2}+O\left(\epsilon^{7 / 2}\right), \frac{z}{z_{0}}=1+\epsilon
\end{array}\right.
\end{aligned}
$$

where now we define $k=|2 a|^{1 / 2}$. This can be inverted to find $z(\rho)$ and $M(\rho)$ to first order in $b$, 


\section{B. Cosmology of codimension one branes in six dimensions}

In section 2 , we mentioned that the case where $z^{\prime}(0) \neq 0$ corresponds to having a 4 -brane rather than a 3-brane at the origin, and that such a model is similar to RS in 5 dimensions. Here we will show this explicitly, following closely the formalism of [37].

We write the metric as

$$
d s^{2}=-n^{2}(r, t) d t^{2}+a^{2}(r, t) d x^{2}+b^{2}(r, t) d r^{2}+c^{2}(r, t) d \theta^{2}
$$

and include a scalar to stabilize the bulk. We will write the branes' stress energy tensors as:

$$
\begin{aligned}
T_{n}^{m}= & \delta(b r) \operatorname{diag}\left(V_{0}+\rho_{*}, V_{0}-p_{*}, V_{0}-p_{*}, V_{0}-p_{*}, 0, V_{0}-p_{*}^{\theta}\right) \\
& +\delta(b(r-R)) \operatorname{diag}\left(V_{1}+\rho, V_{1}-p, V_{1}-p, V_{1}-p, 0, V_{1}-p^{\theta}\right)
\end{aligned}
$$

where the perturbations $\rho, p$ have arbitrary equation of state. We will expand the metric components around the static solution

$$
\begin{aligned}
n(r, t) & =\tilde{a}(r) e^{-N_{1}(r, t)} ; \quad a(r, t)=\tilde{a}(r) a_{0}(t) e^{-A_{1}(r, t)} \\
b(r, t) & =1+B_{1}(r, t) ; \quad c(r, t)=\tilde{a}(r) c_{0}(t) e^{-C_{1}(r, t)} \\
\phi(r, t) & =\phi_{0}(r)+\phi_{1}(r, t)
\end{aligned}
$$

where $\tilde{a}$ and $\phi_{0}$ correspond to the static solutions and the other terms are higher order in powers of $\rho$.

\section{B.1 Boundary conditions}

The complications related to having a codimension-2 brane, and a 4-brane for which $T_{0}^{0} \neq$ $T_{\theta}^{\theta}$ are not present in this model, since we are simply dealing with a space cut off by two codimension- 1 branes. We simply impose $S_{1} / Z_{2}$ symmetry, so that the boundary conditions will be

$$
\begin{aligned}
\left.\left(3 \frac{a^{\prime}}{a}+\frac{c^{\prime}}{c}\right)\right|_{y=R} & =\left.\frac{b(R) \kappa^{2}}{2} T_{0}^{0}\right|_{y=R} \\
\left.\left(2 \frac{a^{\prime}}{a}+\frac{c^{\prime}}{c}+\frac{n^{\prime}}{n}\right)\right|_{y=R} & =\left.\frac{b(R) \kappa^{2}}{2} T_{i}^{i}\right|_{y=R} \\
\left.\left(3 \frac{a^{\prime}}{a}+\frac{n^{\prime}}{n}\right)\right|_{y=R} & =\left.\frac{b(R) \kappa^{2}}{2} T_{5}^{5}\right|_{y=R} \\
\left.\left(3 \frac{a^{\prime}}{a}+\frac{c^{\prime}}{c}\right)\right|_{y=0} & =-\left.\frac{b(0) \kappa^{2}}{2} T_{0}^{0}\right|_{y=0} \\
\left.\left(2 \frac{a^{\prime}}{a}+\frac{c^{\prime}}{c}+\frac{n^{\prime}}{n}\right)\right|_{y=0} & =-\left.\frac{b(0) \kappa^{2}}{2} T_{i}^{i}\right|_{y=0} \\
\left.\left(3 \frac{a^{\prime}}{a}+\frac{n^{\prime}}{n}\right)\right|_{y=0} & =-\left.\frac{b(0) \kappa^{2}}{2} T_{5}^{5}\right|_{y=0} .
\end{aligned}
$$


We must also consider the boundary conditions for the scalar field equation, which are

$$
\begin{aligned}
& \left.\frac{2}{b} \phi^{\prime}\right|_{r=0}=V_{0}^{\prime} \\
& \left.\frac{2}{b} \phi^{\prime}\right|_{r=R}=V_{1}^{\prime}
\end{aligned}
$$

\section{B.2 Order $\left(\rho^{0}\right)$}

The static solutions must solve the Einstein and scalar field equations expanded to zeroth order in $\rho$. These can be written as

$$
\begin{aligned}
\frac{\partial}{\partial \phi_{0}} V\left(\phi_{0}\right) & =\phi_{0}^{\prime \prime}+\phi_{0}^{\prime} 5 \frac{\tilde{a}^{\prime}}{\tilde{a}} \\
V\left(\phi_{0}\right) & =-\Lambda+\frac{1}{2} \phi_{0}^{\prime 2}-10 \kappa^{-2}\left(\frac{\tilde{a}^{\prime}}{\tilde{a}}\right)^{2} \\
-4 \frac{\tilde{a}^{\prime \prime}}{\tilde{a}} & =\kappa^{2}\left(V\left(\phi_{0}\right)+\frac{1}{2} \phi_{0}^{\prime 2}+\Lambda\right)+6\left(\frac{\tilde{a}^{\prime}}{\tilde{a}}\right)^{2}
\end{aligned}
$$

Using the superpotential method [38], one can find an exact solution

$$
\begin{aligned}
\tilde{a}(r) & =\tilde{c}(r)=e^{-A_{0}(r)} \\
A_{0}(r) & =\frac{2}{5} k r+\frac{\kappa^{2} v_{0}^{2}}{16}\left(e^{-2 \epsilon k r}-1\right) \\
\phi_{0} & =v_{0} e^{-\epsilon k r} \\
V\left(\phi_{0}\right) & =\epsilon k^{2} \phi_{0}^{2}\left(1+\frac{\epsilon}{2}-\frac{5}{32} \kappa^{2} \epsilon \phi_{0}^{2}\right)
\end{aligned}
$$

The boundary conditions at lowest order in $\rho$ impose:

$$
\begin{aligned}
& V_{0}=-\frac{10 \Lambda}{k}\left(1-\frac{5}{16} \kappa^{2} v_{0}^{2} \epsilon\right) \\
& V_{1}=\frac{10 \Lambda}{k}\left(1-\frac{5}{16} \kappa^{2} v_{0}^{2} \epsilon e^{-2 \epsilon k R}\right)
\end{aligned}
$$

where

$$
\Lambda=-\frac{8 k^{2}}{5 \kappa^{2}} .
$$

It is clear that at the static level, this model is completely analogous to Randall-Sundrum.

\section{B.3 Order $\rho$}

We now look at the perturbed equations. We first define the following variables:

$$
\begin{aligned}
\Psi_{1} & =-\left(3 A_{1}^{\prime}+C_{1}^{\prime}+4 B_{1} \frac{\tilde{a}^{\prime}}{\tilde{a}}\right)+\kappa^{2} \phi_{0}^{\prime} \phi_{1} \\
\Upsilon_{1} & =N_{1}^{\prime}-A_{1}^{\prime} \\
\xi_{1} & =-\left(3 A_{1}^{\prime}+N_{1}^{\prime}+4 B_{1} \frac{\tilde{a}^{\prime}}{\tilde{a}}\right)+\kappa^{2} \phi_{0}^{\prime} \phi_{1} \\
\zeta_{1} & =\phi_{0}^{\prime \prime} \phi_{1}-\phi_{0}^{\prime} \phi_{1}^{\prime}+\phi_{0}^{\prime 2} B_{1}
\end{aligned}
$$


which appear naturally in the boundary conditions. It is simple to check that these are invariant under the following gauge transformations:

$$
\begin{aligned}
y & =\bar{y}+f(\bar{y}) ; \quad f(0)=f(1)=0 \\
A_{1} & \rightarrow A_{1}-\frac{\tilde{a}^{\prime}}{\tilde{a}} f ; \quad N_{1} \rightarrow N_{1}-\frac{\tilde{a}^{\prime}}{\tilde{a}} f \\
B_{1} & \rightarrow B_{1}+f^{\prime} ; \quad \phi_{1} \rightarrow \phi_{1}+\phi_{0}^{\prime} f \\
C_{1} & \rightarrow C_{1}-\frac{\tilde{a}^{\prime}}{\tilde{a}} f .
\end{aligned}
$$

These new variables obey the boundary conditions

$$
\begin{aligned}
& \left.\Psi_{1}\right|_{y=R}=\frac{\kappa^{2}}{2} \rho ;\left.\quad \Psi_{1}\right|_{y=0}=-\frac{\kappa^{2}}{2} \rho_{*} \\
& \left.\Upsilon_{1}\right|_{y=R}=\frac{\kappa^{2}}{2}(\rho+p) ;\left.\quad \Upsilon_{1}\right|_{y=0}=-\frac{\kappa^{2}}{2}\left(\rho_{*}+p_{*}\right) \\
& \left.\xi_{1}\right|_{y=R}=-\frac{\kappa^{2}}{2} p^{\theta} ;\left.\quad \xi_{1}\right|_{y=0}=\frac{\kappa^{2}}{2} p_{*}^{\theta}
\end{aligned}
$$

Since we still haven't fixed a gauge, we will now choose to work in the stiff potential limit, i.e. $\phi_{1} \equiv 0$ [37]. This allows us to write the first order in $\rho$ terms of the Einstein equations as :

$$
\begin{aligned}
& 3 e^{2 A_{0}}\left[\left(\frac{\dot{a}_{0}}{a_{0}}\right)^{2}+\frac{\dot{a}_{0}}{a_{0}} \frac{\dot{c}_{0}}{c_{0}}\right]_{1}=\Psi_{1}^{\prime}-5 A_{0}^{\prime} \Psi_{1} \\
& e^{2 A_{0}}\left[2\left(\left(\frac{\dot{a}_{0}}{a_{0}}\right)^{2}-\frac{\ddot{a}_{0}}{a_{0}}\right)+\frac{\dot{a}_{0}}{a_{0}} \frac{\dot{c}_{0}}{c_{0}}-\frac{\ddot{c}_{0}}{c_{0}}\right]_{1}=\Upsilon_{1}^{\prime}-5 A_{0}^{\prime} \Upsilon_{1} \\
& 3 e^{2 A_{0}}\left[\frac{\ddot{a}_{0}}{a_{0}}+\left(\frac{\dot{a}_{0}}{a_{0}}\right)^{2}\right]_{1}=\xi_{1}^{\prime}-5 A_{0}^{\prime} \xi_{1} \\
& 3 e^{2 A_{0}}\left[\frac{\ddot{a}_{0}}{a_{0}}+\left(\frac{\dot{a}_{0}}{a_{0}}\right)^{2}+\frac{\dot{a}_{0}}{a_{0}} \frac{\dot{c}_{0}}{c_{0}}+\frac{1}{3} \frac{\ddot{c}_{0}}{c_{0}}\right]_{1}=-A_{0}^{\prime}\left(\xi_{1}+4 \Psi_{1}-3 \Upsilon_{1}\right)+\kappa^{2} \phi_{0}^{\prime 2} B_{1} \\
& \dot{\Psi}_{1}+3\left(\frac{\dot{a}_{0}}{a_{0}}\right)_{1 / 2} \Upsilon_{1}+\left(\frac{\dot{c}_{0}}{c_{0}}\right)_{1 / 2}\left(\Psi_{1}-\xi_{1}\right)=0
\end{aligned}
$$

The solutions are given by:

$$
\begin{aligned}
& \Psi_{1}(r)=e^{5 A_{0}(r)}\left[\left.\Psi\right|_{r=0}+3\left[\left(\frac{\dot{a}_{0}}{a_{0}}\right)^{2}+\frac{\dot{a}_{0}}{a_{0}} \frac{\dot{c}_{0}}{c_{0}}\right]_{1} \int_{0}^{r} e^{-3 A_{0}(r)} d r\right] \\
& \Upsilon_{1}(r)=e^{5 A_{0}(r)}\left[\left.\Upsilon\right|_{r=0}+\left[2\left(\left(\frac{\dot{a}_{0}}{a_{0}}\right)^{2}-\frac{\ddot{a}_{0}}{a_{0}}\right)+\frac{\dot{a}_{0}}{a_{0}} \frac{\dot{c}_{0}}{c_{0}}-\frac{\ddot{c}_{0}}{c_{0}}\right]_{1} \int_{0}^{r} e^{-3 A_{0}(r)} d r\right] \\
& \xi_{1}(r)=e^{5 A_{0}(r)}\left[\left.\xi\right|_{r=0}+3\left[\frac{\ddot{a}_{0}}{a_{0}}+\left(\frac{\dot{a}_{0}}{a_{0}}\right)^{2}\right] \int_{1}^{r} e^{-3 A_{0}(r)} d r\right]
\end{aligned}
$$


The Friedmann equations are then, using $8 \pi G \equiv \frac{\kappa^{2}}{2 \int_{0}^{R} e^{-3 A_{0}(r)} d r}$,

$$
\begin{aligned}
\left(\frac{\dot{a}_{0}}{a_{0}}\right)^{2} & =\frac{8 \pi G}{3}\left[\rho_{*}+e^{-5 A_{0}(R)} \rho\right]-\frac{\dot{a}_{0}}{a_{0}} \frac{\dot{c}_{0}}{c_{0}} \\
\frac{\ddot{a}_{0}}{a_{0}}-\left(\frac{\dot{a}_{0}}{a_{0}}\right)^{2} & =-4 \pi G\left[\rho_{*}+p_{*}+e^{-5 A_{0}(R)}(\rho+p)\right]+\frac{1}{2} \frac{\dot{a}_{0}}{a_{0}} \frac{\dot{c}_{0}}{c_{0}}-\frac{1}{2} \frac{\ddot{c}_{0}}{c_{0}} \\
\frac{\ddot{a}_{0}}{a_{0}}+\left(\frac{\dot{a}_{0}}{a_{0}}\right)^{2} & =-\frac{8 \pi G}{3}\left[\left(p_{*}^{\theta}+e^{-5 A_{0}(R)} p^{\theta}\right]\right.
\end{aligned}
$$

We can combine these to write:

$$
3 \frac{\dot{a}_{0}}{a_{0}} \frac{\dot{c}_{0}}{c_{0}}+\frac{\ddot{c}_{0}}{c_{0}}=\frac{8 \pi G}{3}\left[\rho_{*}-3 p_{*}+e^{-5 A_{0}(R)}(\rho-3 p)+2\left(p_{*}^{\theta}+e^{-5 A_{0}(R)} p^{\theta}\right)\right]
$$

We can then use the fourth Einstein equation to solve for $B_{1}$ :

$$
B_{1}=\frac{1}{6 \phi_{0}^{\prime 2}}\left[\left(\rho_{*}-3 p_{*}-p_{*}^{\theta}\right)\left(F(r)-3 A_{0}^{\prime} e^{5 A_{0}}\right)+e^{-5 A_{0}(R)}\left(\rho-3 p-p^{\theta}\right) F(r)\right]
$$

where we have defined

$$
F=\frac{1}{\int_{0}^{R} e^{-3 A_{0}(r)} d r}\left[e^{2 A_{0}}+3 A_{0}^{\prime} e^{5 A_{0}} \int_{0}^{r} e^{-3 A_{0}(r)} d r\right]
$$

These results are very similar to those one gets in the Randall-Sundrum scenarion. There are however some differences. First, $\rho$ and $p$ denote $(4+1)$-dimensional quantities, since the branes in this model correspond to 4-branes. If the compact dimension is small however, there will be no light Kaluza-Klein modes, and one expects to recover standard cosmology at low energies.

Another difference is the appearance of terms involving the time variation of the compact dimension's scale factor. Such terms are observationally constrained to be quite small, so that they should not lead to appreciable effects on late-time cosmology once a stabilization mechanism for the size of the angular dimension is included [39].

\section{References}

[1] P. Binetruy, C. Deffayet and D. Langlois, "Non-conventional cosmology from a brane-universe," Nucl. Phys. B 565, 269 (2000) [arXiv:hep-th/9905012].

[2] C. Csaki, M. Graesser, C. F. Kolda and J. Terning, "Cosmology of one extra dimension with localized gravity," Phys. Lett. B 462, 34 (1999) [arXiv:hep-ph/9906513].

[3] J. M. Cline, C. Grojean and G. Servant, "Cosmological expansion in the presence of extra dimensions," Phys. Rev. Lett. 83, 4245 (1999) [arXiv:hep-ph/9906523].

[4] V. A. Rubakov and M. E. Shaposhnikov, "Extra spacetime Dimensions: Towards A Solution To The Cosmological Constant Problem," Phys. Lett. B 125, 139 (1983).

[5] C. Wetterich, "The Cosmological Constant And Noncompact Internal Spaces In Kaluza-Klein Theories," Nucl. Phys. B 255, 480 (1985). 
[6] G.W. Gibbons and D.L. Wiltshire, "Spacetime as a membrane in higher dimensions", Nucl. Phys. B 287, 717 (1987).

[7] S. Randjbar-Daemi and C. Wetterich, "Kaluza-Klein Solutions With Noncompact Internal Spaces," Phys. Lett. B 166, 65 (1986).

[8] R. Sundrum, "Compactification for a three-brane universe," Phys. Rev. D 59, 085010 (1999) [arXiv:hep-ph/9807348].

[9] J. W. Chen, M. A. Luty and E. Ponton, "A critical cosmological constant from millimeter extra dimensions," JHEP 0009, 012 (2000) [arXiv:hep-th/0003067].

[10] T. Gherghetta and M. E. Shaposhnikov, "Localizing gravity on a string-like defect in six dimensions," Phys. Rev. Lett. 85, 240 (2000) [arXiv:hep-th/0004014].

[11] G. R. Dvali and G. Gabadadze, "Gravity on a brane in infinite-volume extra space," Phys. Rev. D 63, 065007 (2001) [arXiv:hep-th/0008054].

[12] O. Corradini, A. Iglesias and Z. Kakushadze, "Toward solving the cosmological constant problem?," arXiv:hep-th/0212101.

[13] S. M. Carroll and M. M. Guica, "Sidestepping the cosmological constant with football-shaped extra dimensions," arXiv:hep-th/0302067.

[14] I. Navarro, "Codimension two compactifications and the cosmological constant problem," arXiv:hep-th/0302129.

[15] A. G. Cohen and D. B. Kaplan, "Solving the hierarchy problem with noncompact extra dimensions," Phys. Lett. B 470, 52 (1999) [arXiv:hep-th/9910132].

[16] R. Gregory, "Nonsingular global string compactifications," Phys. Rev. Lett. 84, 2564 (2000) [arXiv:hep-th/9911015].

[17] Z. Chacko and A. E. Nelson, "A solution to the hierarchy problem with an infinitely large extra dimension and moduli stabilization," Phys. Rev. D 62, 085006 (2000) [arXiv:hep-th/9912186].

[18] H. Collins and B. Holdom, "The Randall-Sundrum scenario with an extra warped dimension," Phys. Rev. D 64, 064003 (2001) [arXiv:hep-ph/0103103].

[19] J. E. Kim, B. Kyae and H. M. Lee, "Localized gravity and mass hierarchy in D $=6$ with Gauss-Bonnet term," Phys. Rev. D 64, 065011 (2001) [arXiv:hep-th/0104150].

[20] Z. Chacko, P. J. Fox, A. E. Nelson and N. Weiner, "Large extra dimensions from a small extra dimension," JHEP 0203, 001 (2002) [arXiv:hep-ph/0106343].

[21] P. Kanti, R. Madden and K. A. Olive, "A 6-D brane world model," Phys. Rev. D 64, 044021 (2001) [arXiv:hep-th/0104177].

[22] C. P. Burgess, J. M. Cline, N. R. Constable and H. Firouzjahi, "Dynamical stability of six-dimensional warped brane-worlds," JHEP 0201, 014 (2002) [arXiv:hep-th/0112047].

[23] M. Giovannini, H. Meyer and M. E. Shaposhnikov, "Warped compactification on Abelian vortex in six dimensions," Nucl. Phys. B 619, 615 (2001) [arXiv:hep-th/0104118].

[24] I. I. Kogan, S. Mouslopoulos, A. Papazoglou and G. G. Ross, "Multigravity in six dimensions: Generating bounces with flat positive tension branes," Phys. Rev. D 64, 124014 (2001) [arXiv:hep-th/0107086]. 
[25] O. Corradini, A. Iglesias, Z. Kakushadze and P. Langfelder, "Gravity on a 3-brane in 6D bulk," Phys. Lett. B 521, 96 (2001) [arXiv:hep-th/0108055].

[26] J. Louko and D. L. Wiltshire, "Brane worlds with bolts," JHEP 0202, 007 (2002) [arXiv:hep-th/0109099].

[27] D. K. Park and H. s. Kim, "Single 3-brane brane-world in six dimension," Nucl. Phys. B 650, 114 (2003) [arXiv:hep-th/0206002].

[28] T. Multamaki and I. Vilja, "Warped and compact extra dimensions: 5D branes in 6D models," Phys. Lett. B 545, 389 (2002) [arXiv:hep-th/0207263]. "Cosmological solutions of braneworlds with warped and compact dimensions," Phys. Lett. B 559, 1 (2003) [arXiv:hep-th/0301168].

[29] E. Ponton and E. Poppitz, "Casimir energy and radius stabilization in five and six dimensional orbifolds," JHEP 0106, 019 (2001) [arXiv:hep-ph/0105021].

[30] S. M. Carroll, J. Geddes, M. B. Hoffman and R. M. Wald, "Classical stabilization of homogeneous extra dimensions," Phys. Rev. D 66, 024036 (2002) [arXiv:hep-th/0110149].

[31] Y. Aghababaie, C. P. Burgess, S. L. Parameswaran and F. Quevedo, "SUSY breaking and moduli stabilization from fluxes in gauged 6D supergravity," JHEP 0303, 032 (2003) [arXiv:hep-th/0212091].

[32] U. Gunther, P. Moniz and A. Zhuk, "Nonlinear multidimensional cosmological models with form fields: Stabilization of extra dimensions and the cosmological constant problem," arXiv:hep-th/0303023.

[33] G. T. Horowitz and R. C. Myers, "The AdS/CFT correspondence and a new positive energy conjecture for general relativity," Phys. Rev. D 59, 026005 (1999) [arXiv:hep-th/9808079].

[34] F. Leblond, R. C. Myers and D. J. Winters, "Consistency conditions for brane worlds in arbitrary dimensions," JHEP 0107, 031 (2001) [arXiv:hep-th/0106140].

[35] L. Randall and R. Sundrum, "A large mass hierarchy from a small extra dimension," Phys. Rev. Lett. 83, 3370 (1999) [hep-ph/9905221]; "An alternative to compactification," Phys. Rev. Lett. 83, 4690 (1999) [hep-th/9906064]

[36] W. D. Goldberger and M. B. Wise, "Modulus stabilization with bulk fields," Phys. Rev. Lett. 83, 4922 (1999) [hep-ph/9907447].

[37] J. M. Cline and H. Firouzjahi, "5-dimensional warped cosmological solutions with radius stabilization by a bulk scalar," Phys. Lett. B 495, 271 (2000) [hep-th/0008185], J .M. Cline and J. Vinet, "Order $\rho^{2}$ Corrections to Randall-Sundrum I Cosmology," JHEP 02 (2002) 042, [hep-th/0201041].

[38] O. DeWolfe, D. Z. Freedman, S. S. Gubser and A. Karch, "Modeling the fifth dimension with scalars and gravity," Phys. Rev. D 62, 046008 (2000) [hep-th/9909134].

[39] J. M. Cline and J. Vinet, "Problems with time-varying extra dimensions or 'Cardassian expansion' as alternatives to dark energy," arXiv:hep-ph/0211284.

[40] M. Giovannini, "Gauge field localization on Abelian vortices in six dimensions," Phys. Rev. D 66, 044016 (2002) [arXiv:hep-th/0205139].

[41] M. Giovannini, J. V. Le Be and S. Riederer, "Zero modes of six-dimensional Abelian vortices," Class. Quant. Grav. 19, 3357 (2002) [arXiv:hep-th/0205222]. 
[42] S. Randjbar-Daemi and M. Shaposhnikov, "A formalism to analyze the spectrum of brane world scenarios," Nucl. Phys. B 645, 188 (2002) [arXiv:hep-th/0206016]; "QED from six-dimensional vortex and gauge anomalies," JHEP 0304, 016 (2003)

[arXiv:hep-th/0303247]. 